\title{
目 \\ MINIMIZERS OF CONVEX FUNCTIONALS ARISING IN RANDOM SURFACES
}

\author{
D. DE SILVA AND O. SAVIN
}

\begin{abstract}
We investigate $C^{1}$ regularity of minimizers to $\int F(\nabla u) d x$ in two dimensions for certain classes of non-smooth convex functionals $F$. In particular our results apply to the surface tensions that appear in recent works on random surfaces and random tilings of Kenyon, Okounkov and others.
\end{abstract}

\section{INTRODUCTION}

The classical problem in the calculus of variations consists in minimizing the functional

$$
\min _{\mathcal{A}} \int_{\Omega} F(\nabla u) d x, \quad \Omega \subset \mathbb{R}^{n}
$$

where $F: \mathbb{R}^{n} \rightarrow \mathbb{R}$ is a given convex function and $\mathcal{A}$ is a family of admissible functions $u: \Omega \rightarrow \mathbb{R}$ (typically the elements of a functional space satisfying a boundary condition $u=\varphi$ on $\partial \Omega$ ). In general it is not hard to show the existence and uniqueness for this problem, but the main difficulty arises when trying to prove further smoothness properties of the minimizers. For example, one would like to show that a minimizer has continuous second derivatives. Then it satisfies the Euler-Lagrange equation

$$
\operatorname{div}(\nabla F(\nabla u))=F_{i j}(\nabla u) u_{i j}=0 .
$$

Hilbert's 19th problem posed at the beginning of the last century refers precisely to this question of regularity: Are the solutions of regular problems in the calculus of variations always necessarily analytic?

In two dimensions the problem was solved in 1943 by C.B. Morrey $[\mathrm{M}]$ who showed analyticity of solutions by the use of complex analysis and quasiconformal mappings. A partial answer to Hilbert's problem in the general case was given in the 1930s by the use of Schauder's estimates for linear equations [Sc1], [Sc2], which guarantee that minimizers are smooth once they have Hölder continuous derivatives. On the other hand it follows from the comparison principle that minimizers are Lipschitz continuous under quite general conditions on the domain and boundary data. One piece of the puzzle remained to be proved: that Lipschitz minimizers are in fact $C^{1, \alpha}$.

The breakthrough came in the 1950s from the work of E. De Giorgi D on the minimal surface equation and independently from J. Nash $[\mathrm{N}]$. Differentiating the equation (1.1) with respect to $x_{k}$ we see that the derivative $u_{k}$ satisfies the nonlinear elliptic PDE

$$
\partial_{i}\left(F_{i j}(\nabla u) u_{k j}\right)=0
$$

\footnotetext{
${ }^{1}$ The second author was supported by N.S.F. Grant DMS-07-01037 and a Sloan Fellowship.
} 
Ignoring the dependence on $\nabla u$ we can write that $v=u_{k}$ satisfies the linear equation

$$
\partial_{i}\left(a_{i j}(x) v_{j}\right)=0 .
$$

De Giorgi observed that the uniform ellipticity condition

$$
\lambda I \leq\left[a_{i j}(x)\right]_{i, j} \leq \Lambda I
$$

on the coefficients is sufficient to obtain continuity for $v$ (in fact Hölder continuity due to scaling). The proof is based on an iteration scheme for various Caccioppoli type inequalities of the form

$$
\int_{\Omega}|\nabla v|^{2} \eta^{2} d x \leq C(\Lambda, \lambda) \int_{\Omega} v^{2}|\nabla \eta|^{2} d x, \quad \forall \eta \in C_{0}^{\infty}(\Omega) .
$$

This result led to extensive study of the theory of linear second order elliptic equations with measurable coefficients (see for example [CFMS, [GT], [LSW]).

With the question of regularity being understood in the case when $F$ is smooth and strictly uniformly convex, it is natural to further investigate what happens when the uniform ellipticity condition on $D^{2} F$ fails on a certain set. In the particular case of the $p$-Laplace equation, i.e $F(\xi)=|\xi|^{p}, 1<p<\infty$, when the degeneracy set consists of only one point (the origin) the regularity of minimizers is well-understood (see fore example [E], [U]).

In this paper we direct our attention to the case when the degeneracy set of $F$ can possibly be very large. The first step towards proving regularity of minimizers would be obtaining $C^{1}$ continuity. Then the Euler Lagrange equation becomes less ambiguous and the classical theory can be applied near the points whose gradients lie outside the degeneracy set of $F$.

If $F^{*}$ denotes the Legendre transform of $F$ then

$$
F_{i j}\left(\nabla F^{*}\right) F_{i j}^{*}=\operatorname{tr}\left(\left(D^{2} F^{*}\right)^{-1} D^{2} F^{*}\right)=n,
$$

thus $F^{*}$ is a particular solution of the nonlinear PDE (1.2). Without further evidence we can ask wether or not minimizers have the same regularity as $F^{*}$. In particular: Is it true that if $F$ is strictly convex then Lipschitz minimizers are of class $C^{1}$ ? This seems to be a difficult question but there is some evidence that suggests that the result might be true at least in two dimensions.

In the first part of the paper we prove a priori estimates that answer the question above in two dimensions for two large classes of strictly convex functionals. Our first theorem shows that if $\mathbb{R}^{2}$ can be covered by two open sets $O_{\lambda}, V_{\Lambda}$,

$$
\begin{aligned}
& O_{\lambda} \subset\left\{p \in \mathbb{R}^{2}, D^{2} F(p)>\lambda I\right\}, \\
& V_{\Lambda} \subset\left\{p \in \mathbb{R}^{2}, D^{2} F(p)<\Lambda I\right\},
\end{aligned}
$$

then $\nabla u$ has a uniform modulus of continuity in the interior of $\Omega$.

Theorem 1.1. Let $u$ be a minimizer with $\|\nabla u\|_{L^{\infty}\left(B_{1}\right)} \leq M$. Assume that

$$
\overline{\mathcal{B}}_{M} \subset O_{\lambda} \cup V_{\Lambda} \text {. }
$$

Then in $B_{1 / 2}, \nabla u$ has a uniform modulus of continuity depending on the modulus of convexity $\omega_{F}$ of $F, O_{\lambda}, V_{\Lambda}, M$ and $\|\nabla F\|_{L^{\infty}\left(\mathcal{B}_{M}\right)}$.

In the second theorem we show that if $D^{2} F>0$ except at a finite number of points then the same result holds. 


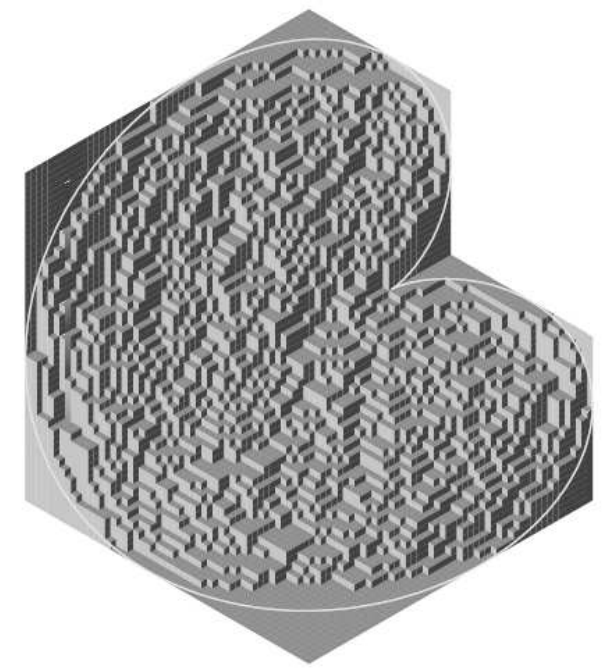

Figure 1.

Theorem 1.2. Let $u$ be a minimizer with $\|\nabla u\|_{L^{\infty}\left(B_{1}\right)} \leq M$. Assume that $\overline{\mathcal{B}}_{M} \backslash$ $\bigcup O_{1 / n}$ is a finite set. Then in $B_{1 / 2}, \nabla u$ has a uniform modulus of continuity depending on $O_{1 / n}, M$ and $\|\nabla F\|_{L^{\infty}\left(\mathcal{B}_{M}\right)}$.

Our interest in this two dimensional regularity problem is motivated by a series of recent papers in combinatorics and statistical mechanics about random tilings and random surfaces by Cohn, Kenyon, Okounkov, Propp, Sheffield and others. Let us briefly explain the connection between our problem and these results.

We present the simplest model of a random surface. For small $\varepsilon$ consider the points $\varepsilon \mathbb{Z}^{3} \subset \mathbb{R}^{3}$, and call the $(1,1,1)$ direction vertical and the plane $P=\{x+$ $y+z=0\}$ horizontal. An $\varepsilon$-stepped surface is a polygonal surface whose faces are squares in the 2 -skeleton of $\varepsilon \mathbb{Z}^{3}$ and which is a graph in the vertical direction. In other words, the subgraph of an $\varepsilon$-stepped surface is a collection of $\varepsilon$-cubes of $\varepsilon \mathbb{Z}^{3}$.

Obviously, stepped surfaces can approximate only Lipschitz graphs with gradients lying in the equilateral triangle $N$ with vertices given by the slopes of the three planes $\{x=0\},\{y=0\},\{z=0\}$.

Consider the "random surfaces" given by all $\varepsilon$ - stepped surfaces above a domain $\Omega \subset P$ which stay $O(\varepsilon)$ away from a given function $\varphi$ defined on $\partial \Omega$. In connection with problems that arise in statistical mechanics, it is interesting to investigate the limiting behavior as $\varepsilon \rightarrow 0$. Cohn, Kenyon and Propp CKP proved that as $\varepsilon \rightarrow 0$ the random surfaces almost surely converge uniformly to a nonrandom function $u$ which solves the variational problem

$$
\min _{u} \int_{\Omega} F(\nabla u) d x, \quad \nabla u \in \bar{N}, \quad u=\varphi \text { on } \partial \Omega .
$$

The surface tension $F: \bar{N} \rightarrow \mathbb{R}$ can be computed from combinatorial considerations and is strictly convex in the interior of $N$ and constant on $\partial N$.

The picture of such a random surface is shown in Figure 1 and it appeared on the cover of the Notices of the AMS, March 2005, Volume 52. 
Other types of random surfaces can be obtained from random tiling of the plane with various geometric figures. For example there is a one to one correspondence between the tilings with $60^{\circ}$ rhombi of an $\varepsilon$ - honeycomb lattice and the $\sqrt{3} \varepsilon$ - stepped surfaces: the tilings are the projections of the faces (squares) along the vertical direction onto the plane $P$.

More generally, one can construct random surfaces corresponding to random perfect matchings (also called dimer configurations) on a weighted, bipartite, periodic, planar graph $G$. Kenyon, Okounkov and Sheffield [KOS] showed that these random surfaces converge a.s. to a solution of (1.4) with $F$ and the polygon $N$ (called the Newton polygon) depending on $G$. The function $F$ can be explicitly computed and minimizers of (1.4) for this $F$ and for a special class of domains and boundary data were studied by Kenyon and Okounkov [KO] using techniques from algebraic geometry.

In particular $F$ is piecewise linear on $\partial N$, strictly convex in the interior, smooth except at a finite number of points where it can have singularities.

In the second part of this paper we investigate precisely the $C^{1}$ regularity of minimizers of (1.4) for an arbitrary functional $F$ which is, in the interior of a polygon $N$, strictly convex and smooth except at a finite number of points, but without any further assumptions about the behavior of $F$ on $\partial N$. In this case the set of degeneracy of $D^{2} F$ is the union of a finite set with $\partial N$.

The variational problem (1.4) is equivalent to an obstacle problem. Indeed, let $\bar{\varphi}(\varphi)$ be the minimum (maximum) of all admissible functions $u$ and define $F$ to be $\infty$ outside $\bar{N}$, then problem (1.4) is equivalent to

$$
\min _{\underline{\varphi} \leq u \leq \bar{\varphi}} \int_{\Omega} F(\nabla u) d x .
$$

The obstacle problem has been extensively studied for the Laplace equation i.e $F(\xi)=|\xi|^{2}$ (see for example $[\mathrm{C}]$ ).

The regularity of minimizers in our case is quite delicate. Near the points where the solution separates from the obstacle the equation becomes degenerate. Moreover, even in the set where $\underline{\varphi}<u<\bar{\varphi}$ it cannot be concluded (as in Laplace equation case) that $\nabla u$ is in the interior of $N$. Thus the results from the first part of the paper cannot be directly applied in any reasonable set.

Our main regularity result says that the minimizers are $C^{1}$ in $\Omega$ except on a number of segments which have an end point on $\partial \Omega$ and have directions perpendicular to the sides of $N$. On these segments the minimizer coincides with either the lower or the upper obstacle.

Theorem 1.3. If $\nabla u$ is discontinuous at $x_{0} \in \Omega$, then there exists a direction $\nu_{i}$ perpendicular to one of the sides $\left[p_{i}, p_{i+1}\right]$ of $N$ such that $u$ is linear on a segment of direction $\nu_{i}$ connecting $x_{0}$ and $\partial \Omega$. Precisely, there exists $x_{1}=x_{0}+t_{1} \nu_{i} \in \partial \Omega$ such that

$$
u(x)=u\left(x_{0}\right)+p_{i} \cdot\left(x-x_{0}\right), \quad \text { for all } x \in\left[x_{0}, x_{1}\right] \subset \bar{\Omega} .
$$

In particular, $u \in C^{1}$ away from the obstacles.

We also prove a type of continuity result at the points where the minimizer is not differentiable. We show that if a sequence of points converges to a point of non-differentiability then their corresponding gradients approach $\partial N$ (see Theorem 4.1). 
As seen in Figure 1, minimizers of (1.4) may have "flat" regions where they are linear and their gradient belongs to the set of vertices of $N$. We show that these regions must lie between a convex and a concave graph (see Theorem 4.2).

The proof of our results relies on two dimensional techniques. For the results in the first part we prove weak versions of Caccioppoli inequalities in which the right hand side of (1.3) is replaced by a constant. This is done using the precise form of the nonlinearity in equation (1.2). As it turns out, such inequalities are sufficient for proving $C^{1}$ continuity. The second part of the paper is more involved. We use the ideas of the first part and an approximation technique together with the fact that any tangent plane splits the graph of a smooth minimizer into at least four connected components. These methods could be exploited further to include other examples of functionals, however most examples would satisfy our assumptions.

As in the work of De Giorgi the key step in proving $C^{1}$ continuity consists in obtaining "localization" lemmas (see Lemmas 2.1, 2.2, 3.1, and Theorem 6.1). Roughly speaking, they say that when we restrict to smaller and smaller domains the image of the gradient restricts itself to either one of two known sets (that decompose the plane).

The paper is organized as follows. In Section 2 and Section 3 we prove respectively Theorem 1.1 and Theorem 1.2. In Section 4 we present the degenerate obstacle problem and state the main results. In Section 5 we introduce the approximation problem. In Section 6 we prove Theorem 1.3. In Section 7 we prove Theorem 4.1] Finally in the last section we prove two flatness theorems Theorems 4.3 and 4.4 that are used in the course of the proof of Theorem 1.3

\section{The Proof of Theorem 1.1 .}

Let $F: \mathbb{R}^{2} \rightarrow \mathbb{R}$ be a smooth strictly convex function, with modulus of convexity $\omega_{F}:(0,+\infty) \rightarrow(0,+\infty)$, i.e.

$$
F(q)-F(p)-\nabla F(p) \cdot(q-p) \geq \omega_{F}(|q-p|), \quad q \neq p .
$$

Let $0<\lambda \leq \Lambda$, and denote by $O_{\lambda}, V_{\Lambda}$ two open sets such that

$$
\begin{aligned}
& O_{\lambda} \subset\left\{p \in \mathbb{R}^{2}, D^{2} F(p)>\lambda I\right\}, \\
& V_{\Lambda} \subset\left\{p \in \mathbb{R}^{2}, D^{2} F(p)<\Lambda I\right\} .
\end{aligned}
$$

Throughout this paper balls (of radius $r$ ) in the $x$-variable space are denoted by $B_{r}$, while balls in the $p$-variable space (the gradient space), are denoted by $\mathcal{B}_{r}$.

Consider the integral functional

$$
I(u)=\int_{B_{1}} F(\nabla u) d x
$$

We prove the following a priori estimate.

Theorem 1.1. Let $u$ be a minimizer to $I(u)$ with $\|\nabla u\|_{L^{\infty}\left(B_{1}\right)} \leq M$. Assume that

$$
\overline{\mathcal{B}}_{M} \subset O_{\lambda} \cup V_{\Lambda} \text {. }
$$

Then in $B_{1 / 2}, \nabla u$ has a uniform modulus of continuity depending on $\omega_{F}, O_{\lambda}, V_{\Lambda}, M$ and $\|\nabla F\|_{L^{\infty}\left(\mathcal{B}_{M}\right)}$. 
2.1. Statement of the localization Lemmas. The proof of Theorem 1.1 relies on the next two Lemmas. Let $\boldsymbol{e}$ be an arbitrary unit vector (direction) in $\mathbb{R}^{2}$ and denote by

$$
\begin{aligned}
& H_{e}^{+}(c):=\left\{p \in \mathbb{R}^{2} \mid p \cdot \boldsymbol{e}>c\right\}, \\
& H_{e}^{-}(c):=\left\{p \in \mathbb{R}^{2} \mid p \cdot \boldsymbol{e}<c\right\}, \\
& S_{e}\left(c_{0}, c_{1}\right):=\left\{p \in \mathbb{R}^{2} \mid c_{0}<p \cdot \boldsymbol{e}<c_{1}\right\},
\end{aligned}
$$

for some constants $c, c_{0}, c_{1} \in \mathbb{R}$, with $c_{0}<c_{1}$.

Lemma 2.1. Let $u$ be a minimizer to $I(u)$ with $\|\nabla u\|_{L^{\infty}\left(B_{1}\right)} \leq M$. Assume that there exist a direction $\boldsymbol{e}$ and constants $c_{0}, c_{1}$ such that

$$
S_{e}\left(c_{0}, c_{1}\right) \cap \nabla u\left(B_{1}\right) \subset O_{\lambda} .
$$

Then, there exists $\delta>0$ depending on $c_{1}-c_{0}, \lambda, M,\|\nabla F\|_{L^{\infty}\left(\mathcal{B}_{M}\right)}$, such that either

$$
\nabla u\left(B_{\delta}\right) \subset H_{e}^{+}\left(c_{0}\right)
$$

or

$$
\nabla u\left(B_{\delta}\right) \subset H_{e}^{-}\left(c_{1}\right) .
$$

Lemma 2.2. Let $u$ be a minimizer to $I(u)$ with $\|\nabla u\|_{L^{\infty}\left(B_{1}\right)} \leq M$. Assume that there exist a direction $\boldsymbol{e}$ and constants $\tilde{c}, \varepsilon$ such that

$$
H_{e}^{+}(\tilde{c}-\varepsilon) \cap \nabla u\left(B_{1}\right) \subset V_{\Lambda} .
$$

Then, there exists $\delta>0$ depending on $\varepsilon, \Lambda, \omega_{F}, M$, such that either

$$
\nabla u\left(B_{\delta}\right) \subset H_{e}^{+}(\tilde{c}-\varepsilon)
$$

or

$$
\nabla u\left(B_{\delta}\right) \subset H_{e}^{-}(\tilde{c}+\varepsilon) .
$$

As observed in the introduction, these Lemmas say that as we restrict to smaller and smaller domains in the $x$-space the image of the gradient restricts itself to either one of two half-planes. The Caccioppoli-type inequalities in the next subsection will be the key tool towards the proof of these Lemmas.

2.2. Caccioppoli-type inequalities. Let $u$ be a smooth minimizer to $I(u)$, then $u$ satisfies the Euler-Lagrange equation

$$
\operatorname{div}(\nabla F(\nabla u))=0 \text { in } B_{1} .
$$

Differentiating the equation in the direction $\boldsymbol{e}$ we obtain

$$
\int_{B_{1}} F_{i j}(\nabla u) u_{e j} \phi_{i} d x=0, \forall \phi \in C_{0}^{\infty}\left(B_{1}\right) .
$$

Let $c_{0}, c_{1} \in \mathbb{R}$ with $c_{0}<c_{1}$ and denote by

$$
G(t):= \begin{cases}0 & t \leq c_{0}, \\ 1 & t \geq c_{1},\end{cases}
$$

a smooth function with bounded slope $\left|G^{\prime}(t)\right|<C$. 
Proposition 2.3. Let $u$ be a minimizer to $I(u)$ with $\|\nabla u\|_{L^{\infty}\left(B_{1}\right)} \leq M$. Assume that there exist a direction $\boldsymbol{e}$ and constants $c_{0}, c_{1}$ such that

$$
S_{e}\left(c_{0}, c_{1}\right) \cap \nabla u\left(B_{1}\right) \subset O_{\lambda} .
$$

Then,

$$
\int_{B_{1 / 2}}\left|\nabla\left(G\left(u_{e}\right)\right)\right|^{2} d x \leq C
$$

for some constant $C$ depending on $c_{0}, c_{1}, \lambda, M,\|\nabla F\|_{L^{\infty}\left(\mathcal{B}_{M}\right)}$.

Proof. In formula (2.10), let us choose as test function $\phi=\xi^{2} G\left(u_{e}\right)$, with $\xi \in$ $C_{0}^{\infty}\left(B_{1}\right), 0 \leq \xi \leq 1$ and $\xi \equiv 1$ on $B_{1 / 2}$. We obtain

$$
\int_{B_{1}} F_{i j}(\nabla u) u_{e j} \xi^{2}\left(G\left(u_{e}\right)\right)_{i} d x=-2 \int_{B_{1}} F_{i j}(\nabla u) u_{e j} \xi \xi_{i} G\left(u_{e}\right) d x .
$$

Now we analyze the left-hand side of (2.14).

$$
\begin{aligned}
L H S & =\int_{B_{1}} F_{i j}(\nabla u) u_{e j} u_{e i} \xi^{2} G^{\prime}\left(u_{e}\right) d x \\
& \geq \lambda \int_{B_{1}}\left|\nabla u_{e}\right|^{2} \xi^{2} G^{\prime}\left(u_{e}\right) d x \geq c \lambda \int_{B_{1}}\left|\nabla\left(G\left(u_{e}\right)\right)\right|^{2} \xi^{2} d x,
\end{aligned}
$$

where in the first inequality in (2.15) we used the assumption (2.12) and the definition of $O_{\lambda}$, while in the second one we used that $G^{\prime}$ is bounded. Thus,

$$
L H S \geq c \lambda \int_{B_{1}}\left|\nabla\left(G\left(u_{e}\right)\right)\right|^{2} \xi^{2} d x .
$$

On the other hand, using integration by parts we have that the right-hand side of (2.14) is given by

$$
\begin{aligned}
R H S & =-2 \int_{B_{1}} \partial_{e}\left(F_{i}(\nabla u)\right) \xi \xi_{i} G\left(u_{e}\right) d x \\
& =2 \int_{B_{1}} F_{i}(\nabla u) \partial_{e}\left(\xi \xi_{i}\right) G\left(u_{e}\right) d x+\int_{B_{1}} F_{i}(\nabla u) \xi \xi_{i} \partial_{e}\left(G\left(u_{e}\right)\right) d x .
\end{aligned}
$$

Thus, for any $\gamma \leq 1$,

$$
|R H S| \leq \gamma \int_{B_{1}}\left|\nabla\left(G\left(u_{e}\right)\right)\right|^{2} \xi^{2} d x+C / \gamma
$$

with $C$ depending on $\|\nabla F\|_{L^{\infty}\left(\mathcal{B}_{M}\right)}$. Combining (2.16) with (2.17) and choosing $\gamma$ sufficiently small we obtain the desired claim.

Proposition 2.4. Let $u$ be a minimizer to $I(u)$ with $\|\nabla u\|_{L^{\infty}\left(B_{1}\right)} \leq M$. Assume that there exist a direction $\boldsymbol{e}$ and constants $\tilde{c}, \varepsilon$ such that

$$
H_{e}^{+}(\tilde{c}-\varepsilon) \cap \nabla u\left(B_{1}\right) \subset V_{\Lambda} .
$$

Then, set $w=\nabla F(\nabla u)$ and $U=(\nabla u)^{-1}\left(H_{e}^{+}(\tilde{c})\right)$,

$$
\int_{B_{1 / 2} \cap U}|\nabla w|^{2} d x \leq C
$$

for some constant $C$ depending on $\tilde{c}, \varepsilon, \Lambda, M$. 
Proof. In formula (2.10) choose as test function $\phi=\xi^{2}\left(u_{e}-(\tilde{c}-\varepsilon)\right)^{+}$with $\xi \in$ $C_{0}^{\infty}\left(B_{1}\right), 0 \leq \xi \leq 1$ and $\xi \equiv 1$ on $B_{1 / 2}$. Set $U^{\varepsilon}:=(\nabla u)^{-1}\left(H_{e}^{+}(\tilde{c}-\varepsilon)\right) \supset U$.

Then,

$$
\int_{U^{\varepsilon}} F_{i j}(\nabla u) u_{e j} u_{e i} \xi^{2} d x=-2 \int_{B_{1}} F_{i j}(\nabla u) u_{e j} \xi \xi_{i}\left(u_{e}-(\tilde{c}-\varepsilon)\right)^{+} d x .
$$

According to assumption (2.18) and the definition of $V_{\Lambda}$, on the set $U^{\varepsilon}$ we have that

$$
F_{i j}(\nabla u) u_{e j} u_{e i}=\left(\nabla u_{e}\right)^{T} \cdot D^{2} F(\nabla u) \cdot \nabla u_{e} \geq \frac{1}{\Lambda}\left|D^{2} F(\nabla u) \cdot \nabla u_{e}\right|^{2} .
$$

Hence, after bounding the right-hand side of (2.20) using Young's inequality we obtain

$$
\int_{U^{\varepsilon}}\left|D^{2} F(\nabla u) \cdot \nabla u_{e}\right|^{2} \xi^{2} d x \leq \gamma \int_{U^{\varepsilon}}\left|D^{2} F(\nabla u) \cdot \nabla u_{e}\right|^{2} \xi^{2} d x+C / \gamma
$$

for some constant $C$ depending on $\Lambda, M, \tilde{c}, \varepsilon$ and any $\gamma>0$. Hence for $\gamma$ small enough we obtain

$$
\int_{B_{1 / 2} \cap U^{\varepsilon}}\left|D^{2} F(\nabla u) \cdot \nabla u_{e}\right|^{2} d x \leq C .
$$

Now, let $\mathbf{f}$ be a direction close to $\boldsymbol{e}, \mathbf{f} \neq \boldsymbol{e}$, such that for some constant $k \in \mathbb{R}$,

$$
\{p \cdot \mathbf{f}=k\} \cap \mathcal{B}_{M} \subset S_{\boldsymbol{e}}(\tilde{c}-\varepsilon, \tilde{c}) .
$$

Then,

$$
H_{\mathbf{f}}^{+}(k) \cap \nabla u\left(B_{1}\right) \subset V_{\Lambda},
$$

and we can repeat the same argument as above with $U^{k}:=(\nabla u)^{-1}\left(H_{\mathbf{f}}^{+}(k)\right) \supset U$ to conclude that

$$
\int_{B_{1 / 2} \cap U^{k}}\left|D^{2} F(\nabla u) \cdot \nabla u_{\mathbf{f}}\right|^{2} d x \leq C .
$$

Combining (2.22) with (2.23) we obtain

$$
\int_{B_{1 / 2} \cap U}\left|D^{2} F(\nabla u) D^{2} u\right|^{2} d x \leq C,
$$

which gives the desired inequality.

2.3. The proof of the localization Lemmas. We are now ready to prove the localization Lemmas, using the Caccioppoli-type inequalities above and the fact that our problem is two dimensional.

Proof of Lemma 2.1. Let $\delta>0$ and assume that there exist $x_{0}, x_{1} \in B_{\delta}$ such that

$$
\nabla u\left(x_{0}\right) \cdot \boldsymbol{e} \leq c_{0}, \quad \nabla u\left(x_{1}\right) \cdot \boldsymbol{e} \geq c_{1} .
$$

Then, by the maximum principle

$$
\begin{aligned}
& \left\{\nabla u \cdot \boldsymbol{e} \leq c_{0}\right\} \cap \partial B_{r} \neq \emptyset, \\
& \left\{\nabla u \cdot \boldsymbol{e} \geq c_{1}\right\} \cap \partial B_{r} \neq \emptyset,
\end{aligned}
$$

for all $r$, with $\delta \leq r \leq 1 / 2$. Let

$$
x_{0}^{r} \in\left\{\nabla u \cdot e \leq c_{0}\right\} \cap \partial B_{r}, \quad x_{1}^{r} \in\left\{\nabla u \cdot e \geq c_{1}\right\} \cap \partial B_{r}
$$


for all $\delta \leq r \leq 1 / 2$. Then, if $G$ is the function defined in (2.11), we have

$$
1=G\left(u_{e}\left(x_{1}^{r}\right)\right)-G\left(u_{e}\left(x_{0}^{r}\right)\right) \leq \int_{\partial B_{r}\left(x_{0}^{r}, x_{1}^{r}\right)}\left|\nabla\left(G\left(u_{e}\right)\right)\right| d s .
$$

Applying Cauchy-Schwartz we obtain

$$
\int_{\partial B_{r}\left(x_{0}^{r}, x_{1}^{r}\right)}\left|\nabla\left(G\left(u_{e}\right)\right)\right|^{2} d s \geq \frac{1}{2 \pi r}
$$

Thus,

$$
\int_{\delta}^{1 / 2} \int_{\partial B_{r}\left(x_{0}^{r}, x_{1}^{r}\right)}\left|\nabla\left(G\left(u_{e}\right)\right)\right|^{2} d s d r \geq \frac{1}{2 \pi} \int_{\delta}^{1 / 2} \frac{1}{r} d r=\frac{1}{2 \pi} \ln \frac{1}{2 \delta}
$$

Hence,

$$
\int_{B_{1 / 2}}\left|\nabla\left(G\left(u_{e}\right)\right)\right|^{2} d x \geq \frac{1}{2 \pi} \ln \frac{1}{2 \delta}
$$

which for $\delta$ sufficiently small depending on $c_{0}, c_{1}, \lambda, M,\|\nabla F\|_{L^{\infty}\left(\mathcal{B}_{M}\right)}$, contradicts the inequality (2.13).

Proof of Lemma 2.2, Let $\delta>0$ and assume that there exist $x_{0}, x_{1} \in B_{\delta}$ such that

$$
\nabla u\left(x_{0}\right) \cdot \boldsymbol{e} \leq \tilde{c}, \quad \nabla u\left(x_{1}\right) \cdot \boldsymbol{e} \geq \tilde{c}+\varepsilon .
$$

Then, as in the previous lemma, for all $r$ with $\delta \leq r \leq 1 / 2$ there are points $x_{0}^{r}, x_{1}^{r} \in \partial B_{r}$ such that

$$
x_{0}^{r} \in\{\nabla u \cdot e \leq \tilde{c}\}, \quad x_{1}^{r} \in\{\nabla u \cdot e \geq \tilde{c}+\varepsilon\} .
$$

Denote by

$$
\begin{gathered}
\underline{H}:=\nabla F(\{p \cdot \boldsymbol{e} \leq \tilde{c}\}) \\
\bar{H}:=\nabla F(\{p \cdot \boldsymbol{e} \geq \tilde{c}+\varepsilon\}) .
\end{gathered}
$$

Set $w=\nabla F(\nabla u)$, then $w$ maps $x_{0}^{r}$ and $x_{1}^{r}$ respectively in $\underline{H}$ and $\bar{H}$.

Now, since $F$ is strictly convex, there exists $\eta=\eta(\varepsilon)>0$ depending on $\omega_{F}$ such that

$$
\left|p_{1}-p_{2}\right| \geq \varepsilon \Rightarrow\left|\nabla F\left(p_{1}\right)-\nabla F\left(p_{2}\right)\right| \geq 2 \eta .
$$

Let $\mathcal{G}$ be a smooth function on $\mathbb{R}^{2}$ with $|\nabla \mathcal{G}| \leq 1$ such that

$$
\mathcal{G}(p)= \begin{cases}0 & p \in \underline{H} \\ \eta & p \in \bar{H}\end{cases}
$$

Notice that $\mathcal{G}$ can be obtained by a mollification of $\min \left\{\eta,(\operatorname{dist}(p, \underline{H})-\gamma)^{+}\right\}$, with $\gamma$ small.

Then, we have

$$
\eta=\mathcal{G}\left(w\left(x_{1}^{r}\right)\right)-\mathcal{G}\left(w\left(x_{0}^{r}\right)\right) \leq \int_{\partial B_{r}\left(x_{0}^{r}, x_{1}^{r}\right)}|\nabla(\mathcal{G}(w))| d s .
$$

Arguing as in the proof of the previous Proposition (see computations following (2.24) $)$, we get

$$
\int_{B_{1 / 2}}|\nabla(\mathcal{G}(w))|^{2} d x \geq \frac{\eta}{2 \pi} \ln \frac{1}{2 \delta} .
$$


However,

$$
\int_{B_{1 / 2}}|\nabla(\mathcal{G}(w))|^{2} d x \leq \int_{B_{1 / 2}}|\nabla \mathcal{G}|^{2}|\nabla w|^{2} d x \leq \int_{B_{1 / 2} \cap(\nabla u)^{-1}\left(S_{e}(\tilde{c}, \tilde{c}+\varepsilon)\right)}|\nabla w|^{2} d x .
$$

Therefore, combining (2.26) with (2.27), we obtain a contradiction to (2.19) as long as $\delta$ is sufficiently small.

2.4. The proof of Theorem 1.1. We wish to prove the following claim:

$\forall \varepsilon>0, \exists \delta>0, \delta=\delta\left(\varepsilon, \omega_{F}, O_{\lambda}, V_{\Lambda}, M,\|\nabla F\|_{L^{\infty}\left(\mathcal{B}_{M}\right)}\right)$ such that $\operatorname{diam}\left\{\nabla u\left(B_{\delta}\right)\right\} \leq \varepsilon$ for any minimizer $u$ to $I(u)$.

Since $O_{\lambda}, V_{\Lambda}$ are open sets covering $\overline{\mathcal{B}}_{M}$, there exists $\varepsilon^{*}>0$ such that any ball $\mathcal{B}_{\varepsilon^{*}}(p) \subset \mathcal{B}_{2 M}$ is either contained in $O_{\lambda}$ or in $V_{\Lambda}$.

Now, let $\varepsilon<\varepsilon^{*}$ and let $P_{N}$ be an $N$-th regular polygon with sides of length $\varepsilon / 2$, such that

$$
\mathcal{B}_{M} \subset P_{N} \subset \mathcal{B}_{2 M} .
$$

Thus $N=N(\varepsilon, M)$. Denote by $p_{i}$ the vertices of $P_{N}$, and let $T_{i}$ be the triangle with vertices $p_{i}, p_{i+1}$ and $p_{i+2}, i=1, \ldots N\left(p_{N+1}=p_{1}, p_{N+2}=p_{2}\right)$. Since the length of the sides of $P_{N}$ is $\varepsilon / 2$, clearly $T_{i}$ is included in a ball $\mathcal{B}_{\varepsilon^{*}}(p) \subset \mathcal{B}_{2 M}$. Thus,

$$
\text { either } T_{i} \subset O_{\lambda} \text { or } T_{i} \subset V_{\Lambda} \text {. }
$$

Without loss of generality we can assume that for some $i=1, \ldots, N$,

$$
T_{i} \cap \nabla u\left(B_{1}\right) \neq \emptyset,
$$

otherwise we can work with the polygon $P_{N} \backslash \bigcup_{i} T_{i}$. Let $[p, q]$ be the closed segment joining two points $p$ and $q$. Denote by $m_{i}$ the middle point of the segments $\left[p_{i}, p_{i+1}\right]$. Also let $\boldsymbol{e}_{i}$ be the direction such that

$$
\left[p_{i}, p_{i+2}\right] \subset\left\{p \cdot \boldsymbol{e}_{i}=l_{i}\right\}, \quad\left[m_{i}, m_{i+1}\right] \subset\left\{p \cdot \boldsymbol{e}_{i}=l_{i}+\gamma\right\},
$$

for some $l_{i}, \gamma$, with $0<\gamma<\varepsilon / 2$. Then according to either Lemma 2.1 or Lemma 2.2 we have that either

$$
\nabla u\left(B_{\delta}\right) \subset H^{-}\left(\boldsymbol{e}_{i}, l_{i}+\gamma\right)
$$

or

$$
\nabla u\left(B_{\delta}\right) \subset H^{+}\left(\boldsymbol{e}_{i}, l_{i}\right),
$$

for some $\delta=\delta\left(\varepsilon, \lambda, \Lambda, \omega_{F}, M,\|\nabla F\|_{L^{\infty}\left(\mathcal{B}_{M}\right)}\right)$.

If the latter holds then immediately $\operatorname{diam}\left\{\nabla u\left(B_{\delta}\right)\right\} \leq \varepsilon$ as desired.

Assume that (2.29) does not hold for any choice of $i$. Then, according to (2.28) we have that

$$
\nabla u\left(B_{\delta^{N}}\right) \subset \widetilde{P}_{N}
$$

where $\widetilde{P}_{N}$ is the polygon with vertices the middle points $m_{i}, i=1, \ldots, N$. Now, we repeat this argument with $\widetilde{P}_{N}$ instead of $P_{N}$ and iterate this a finite number of times till we obtain a polygon of diameter smaller than $\varepsilon$. 


\section{The Proof of Theorem 1.2}

As in the previous section, let $F: \mathbb{R}^{2} \rightarrow \mathbb{R}$ be a smooth strictly convex function, and denote by $O_{1 / n}$ an increasing sequence of open sets such that

$$
O_{1 / n} \subset\left\{p \in \mathbb{R}^{2}, D^{2} F(p)>\frac{1}{n} I\right\} .
$$

Recall that,

$$
I(u)=\int_{B_{1}} F(\nabla u) d x
$$

In this section we prove the following a priori estimate.

Theorem 1.2. Let $u$ be a minimizer to $I(u)$ with $\|\nabla u\|_{L^{\infty}\left(B_{1}\right)} \leq M$. Assume that $\overline{\mathcal{B}}_{M} \backslash \bigcup O_{1 / n}$ is a finite set. Then in $B_{1 / 2}, \nabla u$ has a uniform modulus of continuity depending on the sets $O_{1 / n}, M$ and $\|\nabla F\|_{L^{\infty}\left(\mathcal{B}_{M}\right)}$.

The proof of Theorem 1.2 follows the same strategy as the proof of Theorem 1.1. Precisely, we determine a localization Lemma which allows us to apply an iteration argument and obtain the desired modulus of continuity.

Here is the statement of the localization Lemma.

Lemma 3.1. Let $u$ be a minimizer to $I(u)$ with $\|\nabla u\|_{L^{\infty}\left(B_{1}\right)} \leq M$. Assume that

$$
\nabla u\left(B_{1}\right) \cap \mathcal{B}_{\rho}\left(p_{0}\right)=\emptyset, \quad \mathcal{B}_{4 \rho}\left(p_{0}\right) \cap \overline{\mathcal{B}}_{M} \subset O_{1 / n} .
$$

Then, there exists $\delta>0$ depending on $n, M, \rho,\|\nabla F\|_{L^{\infty}\left(\mathcal{B}_{M}\right)}$, such that either

$$
\nabla u\left(B_{\delta}\right) \subset \mathcal{B}_{4 \rho}\left(p_{0}\right)
$$

or

$$
\nabla u\left(B_{\delta}\right) \cap \mathcal{B}_{3 \rho}\left(p_{0}\right)=\emptyset .
$$

In order to prove Lemma 3.1, we use the following preliminary Lemma.

Lemma 3.2. Let $u$ be a minimizer to $I(u)$ in $B_{2}$ such that

$$
\nabla u\left(B_{1}\right) \cap \mathcal{B}_{\varepsilon}\left(p_{0}\right)=\emptyset, \quad \nabla u\left(\partial B_{1}\right) \cap \mathcal{B}_{\delta}\left(p_{0}\right)=\emptyset,
$$

with $0<\varepsilon<\delta$. Then,

$$
\nabla u\left(B_{1}\right) \cap \mathcal{B}_{\delta}\left(p_{0}\right)=\emptyset .
$$

Proof. For simplicity, we take $p_{0}=0$.

In formula (2.10), let us choose $\boldsymbol{e}=e_{k}$ and as test function $\phi=\eta_{k}(\nabla u)$, with $\eta$ being 0 outside the ball $\mathcal{B}_{\delta}$. Notice that $\phi$ is an admissible test function in view of our hypothesis. We obtain,

$$
\int_{B_{1}} F_{i j}(\nabla u) u_{k j}\left(\eta_{k}(\nabla u)\right)_{i} d x=0
$$

Hence differentiating and then summing over all $k$ 's, we get

$$
\int_{B_{1}} \operatorname{Tr}\left(D^{2} F D^{2} u D^{2} \eta D^{2} u\right) d x=0 .
$$

To estimate the integrand above, we use the characteristic polynomial equation for a $2 \times 2$ matrix,

$$
A^{2}-(\operatorname{Tr} A) A+(\operatorname{det} A) I=0 .
$$


In particular,

$$
\operatorname{Tr} A=0 \Rightarrow A^{2}=-(\operatorname{det} A) I .
$$

Thus, since $u$ solves the Euler-Lagrange equation (2.9), we can apply this identity to $A=D^{2} F D^{2} u$ to obtain,

$$
\begin{aligned}
\operatorname{Tr}\left(D^{2} F D^{2} u D^{2} \eta D^{2} u\right) & =\operatorname{Tr}\left(\left(D^{2} F D^{2} u\right)^{2} D^{2} \eta\left(D^{2} F\right)^{-1}\right) \\
& =\operatorname{det} D^{2} F\left|\operatorname{det} D^{2} u\right| \operatorname{Tr}\left(\left(D^{2} F\right)^{-1} D^{2} \eta\right)
\end{aligned}
$$

where in the first equality we used that

$$
\operatorname{Tr}(A B)=\operatorname{Tr}(B A) .
$$

We choose $\eta$ so that

$$
\operatorname{Tr}\left(\left(D^{2} F\right)^{-1} D^{2} \eta\right)>0 \quad \text { in } \mathcal{B}_{\delta} \backslash \overline{\mathcal{B}}_{\varepsilon / 2} .
$$

For example, for $\delta=1$

$$
\eta(p)= \begin{cases}e^{-k|p|}-k e^{-k}|p|+(k-1) e^{-k} & |p| \leq 1 \\ 0 & |p|>1\end{cases}
$$

with $k$ a large enough constant depending on the ellipticity constants for $D^{2} F$ on $\mathcal{B}_{\delta}$. Combining (3.5) with (3.7), we get that

$$
\operatorname{det} D^{2} u=0, \quad \text { on }(\nabla u)^{-1}\left(\mathcal{B}_{\delta} \backslash \overline{\mathcal{B}}_{\varepsilon / 2}\right) \cap B_{1} .
$$

Since $u$ solves the Euler-Lagrange equation we obtain that $D^{2} u=0$ and $\nabla u$ is constant on the set above. Hence this set is both open and closed in $B_{1}$, therefore it is either empty (and we are done) or it coincides with $B_{1}$. In the latter case, we conclude by continuity that also $\nabla u\left(\partial B_{1}\right) \cap \mathcal{B}_{\delta} \neq \emptyset$ and we reach a contradiction.

Next we obtain a Caccioppoli-type inequality.

Proposition 3.3. Let $u$ be a minimizer to $I(u)$ with $\|\nabla u\|_{L^{\infty}\left(B_{1}\right)} \leq M$. Then,

$$
\int_{B_{1 / 2} \cap(\nabla u)^{-1}\left(O_{1 / n}\right)}\left|D^{2} u\right|^{2} d x \leq C
$$

for some constant $C$ depending on $n, M,\|\nabla F\|_{L^{\infty}\left(\mathcal{B}_{M}\right)}$.

Proof. In formula (2.10), let us choose $\boldsymbol{e}=e_{k}$ and as test function $\phi=\xi^{2} F_{k}(\nabla u)$, with $\xi \in C_{0}^{\infty}\left(B_{1}\right), 0 \leq \xi \leq 1$ and $\xi \equiv 1$ on $B_{1 / 2}$. We obtain, after summing over all $k$ 's,

$$
\int_{B_{1}} F_{i j}(\nabla u) u_{k j} \xi^{2}\left(F_{k}(\nabla u)\right)_{i} d x=-2 \int_{B_{1}} F_{i j}(\nabla u) u_{k j} \xi \xi_{i} F_{k}(\nabla u) d x .
$$

Now we analyze the left-hand side of (3.10). We use again (3.6) for $A=D^{2} F D^{2} u$ and we obtain

$$
\begin{aligned}
L H S & =\int_{B_{1}} F_{i j}(\nabla u) u_{k j} \xi^{2} F_{k l}(\nabla u) u_{l i} d x=\int_{B_{1}} \xi^{2} \operatorname{Tr}\left(\left(D^{2} F D^{2} u\right)^{2}\right) d x \\
& =2 \int_{B_{1}} \xi^{2}\left(\operatorname{det} D^{2} F\right)\left|\operatorname{det} D^{2} u\right| d x .
\end{aligned}
$$

Moreover, again using (3.6) we have that

$$
\left|D^{2} u\right|^{2} \leq\left|\left(D^{2} F\right)^{-1}\right|^{2}\left|D^{2} F D^{2} u\right|^{2}=\left|\left(D^{2} F\right)^{-1}\right|^{2}\left|\operatorname{det}\left(D^{2} F D^{2} u\right)\right|
$$


and hence,

$$
\operatorname{det} D^{2} F\left|\operatorname{det} D^{2} u\right| \geq c\left|D^{2} u\right|^{2} \quad \text { on }(\nabla u)^{-1}\left(O_{1 / n}\right)
$$

for some constant $c$ depending on $n$. Thus,

$$
L H S \geq c \int_{B_{1 / 2} \cap(\nabla u)^{-1}\left(O_{1 / n}\right)}\left|D^{2} u\right|^{2} d x
$$

with $c$ depending on $n$.

On the other hand, using integration by parts together with the Euler-Lagrange equation $\partial_{k}\left(F_{k}(\nabla u)\right)=0$, we have that the right-hand side of (3.10) is given by

$$
\begin{aligned}
R H S & =-2 \int_{B_{1}} \partial_{k}\left(F_{j}(\nabla u)\right) \xi \xi_{i} F_{k}(\nabla u) d x \\
& =2 \int_{B_{1}} F_{j}(\nabla u) \partial_{k}\left(\xi \xi_{i}\right) F_{k}(\nabla u) d x,
\end{aligned}
$$

and hence

$$
|R H S| \leq C
$$

with $C$ depending on $\|\nabla F\|_{L^{\infty}\left(\mathcal{B}_{M}\right)}$. Combining (3.10), 3.11) and (3.12) we obtain the desired claim.

Finally, we are ready to exhibit the proof of the localization Lemma 3.1

Proof of Lemma 3.1. Let $\delta>0$ and assume that there exist $x_{0}, x_{1} \in B_{\delta}$ such that

$$
\left|\nabla u\left(x_{0}\right)-p_{0}\right| \geq 4 \rho, \quad\left|\nabla u\left(x_{1}\right)-p_{0}\right|<3 \rho .
$$

Then, by the maximum principle for each $\delta \leq r \leq 1 / 2$ there exists a point $x_{0}^{r} \in \partial B_{r}$ such that

$$
\left|\nabla u\left(x_{0}^{r}\right)-p_{0}\right| \geq 4 \rho .
$$

Also, from Lemma 3.2, for each $\delta \leq r \leq 1 / 2$ there exists a point $x_{1}^{r} \in \partial B_{r}$ such that

$$
\left|\nabla u\left(x_{1}^{r}\right)-p_{0}\right|<3 \rho .
$$

Let $\mathcal{G}$ be a smooth function on $\mathbb{R}^{2}$ with $|\nabla \mathcal{G}| \leq 1 / \rho$ such that

$$
\mathcal{G}(p)= \begin{cases}0 & \left|p-p_{0}\right| \geq 4 \rho, \\ 1 & \left|p-p_{0}\right| \leq 3 \rho .\end{cases}
$$

We proceed as in Lemma 2.2 to obtain,

$$
\int_{B_{1 / 2}}|\nabla(\mathcal{G}(\nabla u))|^{2} d x \geq \frac{1}{2 \pi} \ln \frac{1}{2 \delta} .
$$

However, since $\mathcal{B}_{4 \rho}\left(p_{0}\right) \cap \overline{\mathcal{B}}_{M} \subset O_{1 / n}$, by the definition of $\mathcal{G}$ we get

$$
\int_{B_{1 / 2}}|\nabla(\mathcal{G}(\nabla u))|^{2} d x \leq \int_{B_{1 / 2}}|\nabla \mathcal{G}|^{2}\left|D^{2} u\right|^{2} d x \leq \frac{1}{\rho^{2}} \int_{B_{1 / 2} \cap(\nabla u)^{-1}\left(O_{1 / n}\right)}\left|D^{2} u\right|^{2} d x
$$

Therefore, combining (3.13) with (3.14), we obtain a contradiction to the Caccioppoli inequality (3.9) as long as $\delta$ is sufficiently small. 
We now combine all the ingredients above and provide the proof of Theorem 1.2.

Proof of Theorem 1.2. We wish to prove the following claim:

$\forall \varepsilon>0, \exists \delta>0, \delta=\delta\left(\varepsilon, O_{1 / n}, M,\|\nabla F\|_{L^{\infty}\left(\mathcal{B}_{M}\right)}\right)$ such that $\operatorname{diam}\left\{\nabla u\left(B_{\delta}\right)\right\} \leq \varepsilon$ for any minimizer $u$ to $I(u)$.

Fix $\varepsilon>0$. Let $\overline{\mathcal{B}}_{M} \backslash \bigcup O_{1 / n}=\left\{q_{1}, \ldots, q_{m}\right\}$. We cover the set $\mathcal{B}_{2 M} \backslash \bigcup_{i=1}^{m} \mathcal{B}_{5 \rho}\left(q_{i}\right)$ with a finite number of balls $\mathcal{B}_{\rho}^{k}$ of radius $\rho$, with $\rho$ small, say $\rho=\varepsilon / 5$. Notice that $\mathcal{B}_{4 \rho}^{k} \cap \overline{\mathcal{B}}_{M} \subset O_{1 / n}$, for a large $n$.

Clearly, since $\nabla u\left(B_{1}\right) \subset \overline{\mathcal{B}}_{M}$, then $\nabla u\left(B_{1}\right) \cap \mathcal{B}_{\rho}^{k}=\emptyset$ for some $\mathcal{B}_{\rho}^{k} \subset \mathcal{B}_{2 M} \backslash \overline{\mathcal{B}}_{M}$. Then, according to Lemma 3.1 we have that either

$$
\nabla u\left(B_{\delta}\right) \subset \mathcal{B}_{4 \rho}^{k}
$$

or

$$
\nabla u\left(B_{\delta}\right) \cap \mathcal{B}_{3 \rho}^{k}=\emptyset
$$

for some $\delta>0$. If (3.15) occurs, then we reached our conclusion. Otherwise, if (3.16) occurs, we conclude that $\nabla u\left(B_{\delta}\right) \cap \mathcal{B}_{\rho}^{j}=\emptyset$ for all $\mathcal{B}_{\rho}^{j}$ such that $\mathcal{B}_{\rho}^{j} \cap \mathcal{B}_{\rho}^{k} \neq \emptyset$. Hence we can apply again Lemma 3.1 to the balls $\mathcal{B}_{\rho}^{j}$. We iterate this argument. If at some step we reach the conclusion (3.15), then we are done. If at each step we reach the conclusion (3.16), then after a finite number of steps (because our balls cover a connected domain) we obtain that for some small $\tilde{\delta}, \nabla u\left(B_{\tilde{\delta}}\right) \cap \mathcal{B}_{\rho}^{k}=\emptyset$ for all $k$. Since $\nabla u\left(B_{\tilde{\delta}}\right)$ is connected, we conclude that $\nabla u\left(B_{\tilde{\delta}}\right) \subset \mathcal{B}_{5 \rho}\left(q_{i}\right)$ for some $i$. Again we reach the desired conclusion.

\section{A degenerate obstacle Problem}

4.1. The statement of the problem and preliminaries. Let $N$ be a convex (open) polygon in $\mathbb{R}^{2}$ with $n$ vertices $\left\{p_{1}, \ldots, p_{n}\right\}=\mathcal{P}$ (also, set $p_{n+1}=p_{1}$.) Let $[p, q]$ be the closed segment joining two points $p$ and $q$ and let $(p, q)$ be the open segment joining them. Denote by $\nu_{i}$ a direction perpendicular to the side $\left[p_{i}, p_{i+1}\right], i=1, \ldots, n$. Finally, let $\mathcal{Q}=\left\{q_{1}, \ldots, q_{m}\right\}$ be a finite subset of $N$.

Let $F: \bar{N} \rightarrow \mathbb{R}$ be a convex function such that

(i) $F \in C^{2}(N \backslash \mathcal{Q}), \quad D^{2} F>0$ on $N \backslash \mathcal{Q}$;

(ii) $F$ is bounded.

Let $\Omega \subset \mathbb{R}^{2}$ be a bounded Lipschitz domain, and let $\varphi: \partial \Omega \rightarrow \mathbb{R}$ be a function that admits an extension $\tilde{\varphi}$ with $\nabla \tilde{\varphi} \in \bar{N}$.

We consider the following problem $(P)$ : minimize the functional

$$
I(u)=\int_{\Omega} F(\nabla u) d x
$$

among all Lipschitz competitors $u$, with $u=\varphi$ on $\partial \Omega, \nabla u \in \bar{N}$ (we think of $F$ to be equal to $+\infty$ outside $\bar{N}$.) 
Denote by,

$$
\begin{gathered}
\underline{\varphi}=\inf \{v: v=\varphi \text { on } \partial \Omega, \nabla v \in \bar{N}\} \\
\bar{\varphi}=\sup \{v: v=\varphi \text { on } \partial \Omega, \nabla v \in \bar{N}\} .
\end{gathered}
$$

We refer to $\underline{\varphi}, \bar{\varphi}$ as to respectively, the lower obstacle and upper obstacle.

Also, in what follows we want to distinguish when gradients are close or not to $\partial N$. One way of doing this is to consider the compactification of $N$ with one point. We adopt a slightly different approach by introducing the following function

$$
H: \bar{N} \rightarrow S^{2}
$$

such that, $H$ is continuous, $\partial N$ is mapped to a point, and $H$ is a homeomorphism between $N$ and $H(N)$.

Remark. Notice that our minimization problem is equivalent to the following obstacle problem: minimizing

$$
\widetilde{I}(u)=\int_{\Omega} \widetilde{F}(\nabla u) d x
$$

among all competitors $v$ such that $\underline{\varphi} \leq v \leq \bar{\varphi}$, with $\widetilde{F}$ a convex extension of $F$ outside $\bar{N}$.

4.2. The main results. We state now our main results. Let $u$ be the unique minimizer to the problem $(P)$.

Our first main result says that the minimizers are $C^{1}$ in $\Omega$ except on a number of segments which have an end point on $\partial \Omega$ and have directions perpendicular to the sides of $N$. On these segments the minimizer coincides with one of the lower or upper obstacle.

Theorem 1.3. If $\nabla u$ is discontinuous at $x_{0} \in \Omega$, then there exists a direction $\nu_{i}$ perpendicular to one of the sides $\left[p_{i}, p_{i+1}\right]$ such that $u$ is linear on a segment of direction $\nu_{i}$ connecting $x_{0}$ and $\partial \Omega$. Precisely, there exists $x_{1}=x_{0}+t_{1} \nu_{i} \in \partial \Omega$ such that

$$
u(x)=u\left(x_{0}\right)+p_{i} \cdot\left(x-x_{0}\right), \quad \text { for all } x \in\left[x_{0}, x_{1}\right] \subset \bar{\Omega} .
$$

In particular, $u \in C^{1}$ away from the obstacles.

Our second main result says that if a sequence of points converges to a point of non-differentiability of $u$ then their corresponding gradients approach $\partial N$. Let $H$ be as in (4.1); the precise statement of our result reads as follows.

Theorem 4.1. $H(\nabla u)$ is continuous in $\Omega$.

Minimizers of $(P)$ may develop "flat" regions where they are linear and their gradient belongs to the set of vertices of $N$. The next theorem describes the shape of these regions.

Theorem 4.2. Let $p_{i}$ be a vertex of $N$ and let $\omega_{i}$ be a direction so that

$$
\omega_{i} \cdot\left(p-p_{i}\right)>0 \quad \forall p \in \bar{N} \backslash\left\{p_{i}\right\} .
$$


Let $S$ be the interior of the set $\left\{u(x)=c+p_{i} \cdot x\right\}$ and assume that $S \neq \emptyset$. Then, $\partial S \cap \Omega$ consists of a convex graph (by above) and a concave graph (by below)in the $\omega_{i}$ direction.

Finally, the next two results deal with the case when $u$ is a perturbation of a linear function. They will be used in the proof of our main Theorem 1.3.

Theorem 4.3. Assume $B_{1} \subset \Omega$ and

$$
\mathcal{B}_{\delta}\left(p_{0}\right) \subset N .
$$

Then, there exists $\varepsilon$ depending on $\delta, p_{0}, F$, such that if

$$
\left|u-p_{0} \cdot x\right| \leq \varepsilon, \quad x \in B_{1}
$$

then $u \in C^{1}\left(B_{1 / 2}\right)$ and

$$
\nabla u\left(B_{1 / 2}\right) \subset \mathcal{B}_{\delta}\left(p_{0}\right) .
$$

Theorem 4.4. Assume $B_{1} \subset \Omega$ and let $p_{0} \in \partial N$. Then, there exists $\varepsilon$ depending on $\delta, F$, such that if

$$
\left|u-p_{0} \cdot x\right| \leq \varepsilon, \quad x \in B_{1}
$$

then

$$
\left[\nabla u(x), p_{0}\right] \quad \text { is in a } \delta \text {-neighborhood of } \partial N \text {, for a.e } x \in B_{1 / 2} \text {. }
$$

We finish this section by showing that minimizers to the problem $(P)$ are unique. We use the abbreviation L.P. for Lebesgue point.

Proposition 4.5. The minimization problem $(P)$ admits a unique solution.

Proof. Let $u_{1}, u_{2}$ be two distinct solutions. By the convexity of $F$, we have that $\left(u_{1}+u_{2}\right) / 2$ is also a solution and

$$
\frac{F\left(\nabla u_{1}\right)+F\left(\nabla u_{2}\right)}{2}=F\left(\frac{\nabla u_{1}+\nabla u_{2}}{2}\right), \quad \text { a.e. in } \Omega .
$$

Hence, since $F$ is strictly convex in $N$, we have that if $x$ is a L.P. for $\nabla u_{1}$ and $\nabla u_{2}$ then either

$$
\nabla u_{1}(x)=\nabla u_{2}(x)
$$

or

$$
\nabla u_{1}(x), \nabla u_{2}(x) \in\left[p_{i}, p_{i+1}\right], \quad \text { for some } i=1, \ldots, n \text {. }
$$

Now, let us assume by contradiction that $u_{1}(0)>u_{2}(0), 0 \in \Omega$, say $u_{1}(0)-$ $u_{2}(0)=\varepsilon$. Then, since $u_{1}, u_{2}$ are Lipschitz and they coincide on the boundary, there exists $\rho=c \varepsilon$ such that if $|\tau|<\rho$, then

$$
B_{\rho} \subset\left\{u_{2}(x+\tau)+\varepsilon / 2<u_{1}\right\} \subset \subset \Omega .
$$

The minimality of $u_{2}(x+\tau)$ together with the inclusion above imply that

$$
v_{\tau}(x)=\min \left\{u_{1}(x), u_{2}(x+\tau)+\varepsilon / 2\right\}
$$

is also a minimizer of the problem $(P)$.

Thus, if $x_{1} \in B_{\rho / 2}$ is a L.P. for $\nabla u_{1}$ and $x_{2} \in B_{\rho / 2}$ is a L.P. for $\nabla u_{2}$, we can translate $x_{2}$ by $\tau$ to coincide with $x_{1}$, so that $x_{1}$ is a L.P. for $v_{\tau}$ and according to (4.2)-(4.3) we have either

$$
\nabla u_{1}\left(x_{1}\right)=\nabla u_{2}\left(x_{2}\right)
$$


or

$$
\nabla u_{1}\left(x_{1}\right), \nabla u_{2}\left(x_{2}\right) \in\left[p_{i}, p_{i+1}\right], \quad \text { for some } i=1, \ldots, n \text {. }
$$

Similarly, by appropriate translations we obtain that if $x_{1} \in B_{\rho / 2}$ is a L.P. for $\nabla u_{1}$ and $x_{2}, x_{3}, x_{4} \in B_{\rho / 2}$ are L.P. for $\nabla u_{2}$ then

$$
\begin{aligned}
& F\left(\frac{1}{4}\left(\nabla u_{1}\left(x_{1}\right)+\nabla u_{2}\left(x_{2}\right)+\nabla u_{2}\left(x_{3}\right)+\nabla u_{2}\left(x_{4}\right)\right)\right) \\
& =\frac{1}{4}\left(F\left(\nabla u_{1}\left(x_{1}\right)\right)+F\left(\nabla u_{2}\left(x_{2}\right)\right)+F\left(\nabla u_{2}\left(x_{3}\right)\right)+F\left(\nabla u_{2}\left(x_{4}\right)\right)\right) .
\end{aligned}
$$

Hence

$$
\nabla u_{1}\left(x_{1}\right), \nabla u_{2}\left(x_{2}\right), \nabla u_{2}\left(x_{3}\right), \nabla u_{2}\left(x_{4}\right) \text { are collinear. }
$$

We distinguish two cases.

Case 1. There exists at least one L.P. $\bar{x} \in B_{\rho / 2}$ for either $\nabla u_{1}$ or $\nabla u_{2}$ such that $\bar{p}=\nabla u_{i}(\bar{x}) \in N$. Then, we conclude that $\nabla u_{1}=\nabla u_{2}=\bar{p}$ in $B_{\rho / 2}$. Hence $u_{1}-u_{2}=\varepsilon$ on $B_{\rho / 2}$. Now we can proceed as above with 0 replaced by any point in $B_{\rho / 2}$. By iterating this argument a finite number of times we conclude that $\left\{u_{1}-u_{2}=\varepsilon\right\}$ must coincide with $\Omega$. This contradicts that $u_{1}=u_{2}$ on $\partial \Omega$.

Case 2. All Lebesgue points $x \in B_{\rho / 2}$ for $\nabla u_{i}, i=1,2$ are mapped on $\partial N$.

First we claim that all Lebesgue points $x \in B_{\rho / 2}$ for $\nabla u_{i}, i=1,2$ are mapped on the same side. Indeed, according to (4.6) any three such points for $\nabla u_{2}$ are collinear. The claim follows by interchanging $u_{2}$ and $u_{1}$.

Thus all Lebesgue points of $\nabla u_{1}, \nabla u_{2}$ in $B_{\rho / 2}$ must lie on the same side, say $\left[p_{1}, p_{2}\right]$. Therefore $u_{1}-u_{2}$ is constant $\varepsilon$ in $B_{\rho / 2}$ on the segment with middle point at zero, in the direction perpendicular to $\left[p_{1}, p_{2}\right]$. This implies that the function $\nu \cdot x$ cannot achieve a maximum (minimum) on the set $\left\{u_{1}-u_{2}=\varepsilon\right\}$, where $\nu$ is a direction which differs from any of the perpendicular directions to the sides $\left[p_{i}, p_{i+1}\right]$. This contradicts that $\Omega$ is bounded.

\section{The APPROXimation.}

The goal of this section is to obtain smooth approximations $u_{m}$ that converge uniformly to the minimizer $u$ of the problem $(P)$. Moreover, for any compact $K \Subset \Omega$ we want $\nabla u_{m}(K)$ to lie in any neighborhood of $\bar{N}$ for $m$ large enough. One way of achieving this is to approximate $F$ with smooth convex functions $F_{m}$ that converge to $\infty$ outside $\bar{N}$ and have cubic growth at $\infty$.

Let $\bar{F}$ be a convex function on $\mathbb{R}^{2}, \bar{F}=0$ on $N, \bar{F}(p)=|p|^{3}$ for $|p|$ large, $\bar{F} \in C^{\infty}\left(\mathbb{R}^{2} \backslash \bar{N}\right)$ with $D^{2} \bar{F}>0$ on $\mathbb{R}^{2} \backslash \bar{N}$.

Let $F_{m} \in C^{\infty}\left(\mathbb{R}^{2}\right)$ with $D^{2} F_{m}>0$, be such that

(i) $F_{m} \rightarrow F$ uniformly on $\bar{N}$;

(ii) $D^{2} F_{m} \rightarrow D^{2} F$ uniformly on compacts on $N \backslash \mathcal{Q}$;

(iii) $F_{m}(p)=C_{m} \bar{F}$ in $D_{m}:=\left\{p \in \mathbb{R}^{2} \mid \bar{F}(p)>1 / m\right\}$, with $C_{m} \rightarrow+\infty$. 
Let $u_{m}$ be the minimizer to

$$
\int_{\Omega} F_{m}(\nabla v) d x, \quad v=\varphi \quad \text { on } \partial \Omega .
$$

Here $\varphi$ is the boundary data of the minimizer of the problem $(P)$.

We will show that the $u_{m}$ 's are the desired smooth approximations. Using the lower obstacle $\underline{\varphi}$ as competitor, we see that $\int_{\Omega} F_{m}\left(\nabla u_{m}\right) d x$ is bounded above by a fixed constant. Hence, since $C_{m}>1$ we conclude

$$
\int_{\Omega}\left|\nabla u_{m}\right|^{3} d x \leq C .
$$

By Sobolev embedding theorem we have that $u_{m}$ is uniformly Hölder continuous in $\bar{\Omega}$. Thus, by Ascoli-Arzela there exists a function $u$ such that (for a subsequence of m's)

$$
u_{m} \rightarrow u \text { uniformly on } \bar{\Omega} \text {. }
$$

First we show the following Proposition (recall the definition (4.1) of $H$ from the previous section, and assume $H$ to be defined on the whole $\mathbb{R}^{2}$ and to be constant outside $N)$.

Proposition 5.1. $u$ is the minimizer to the problem $(P)$. Also

$\nabla u_{m} \rightarrow \nabla u$ in measure on $A:=\{x \mid x$ is a L.P. for $\nabla u$ and $\nabla u(x) \in N\}$, and

$$
H\left(\nabla u_{m}\right) \rightarrow H(\nabla u) \text { in measure on } \Omega \text {. }
$$

Proof. Let $v$ be a function on $\bar{\Omega}, v=\varphi$ on $\partial \Omega$ and $\nabla v \in \bar{N}$. Let $\widetilde{F}$ be a convex function on $\mathbb{R}^{2}$ with bounded gradient which approximates $F$ in $N$. Then for $m$ large,

$$
\begin{aligned}
\int_{\Omega} F(\nabla v) d x & \geq \int_{\Omega} F_{m}(\nabla v) d x-\varepsilon \geq \int_{\Omega} F_{m}\left(\nabla u_{m}\right) d x-\varepsilon \geq \int_{\Omega} \widetilde{F}\left(\nabla u_{m}\right) d x-2 \varepsilon \\
& \geq \int_{\Omega}\left(\widetilde{F}(\nabla u)+\nabla \widetilde{F}(\nabla u)\left(\nabla u_{m}-\nabla u\right)+\omega_{\widetilde{F}}\left(\nabla u, \nabla u_{m}-\nabla u\right)\right) d x-2 \varepsilon
\end{aligned}
$$

with $\omega_{\widetilde{F}} \geq 0$ the modulus of convexity of $\widetilde{F}$. Using that $\nabla u_{m} \rightarrow \nabla u$ weakly in $L^{3}$, we conclude that

$$
\int_{\Omega} F(\nabla v) d x \geq \int_{\Omega} \widetilde{F}(\nabla u) d x-2 \varepsilon .
$$

Since $\widetilde{F}$ is arbitrary outside $\bar{N}$, we deduce that $\nabla u \in \bar{N}$ and $u$ is the minimizer of the problem $(P)$.

Since $F$ is strictly convex in $N$ and

$$
\int_{\Omega} \omega_{\widetilde{F}}\left(\nabla u, \nabla u_{m}-\nabla u\right) d x \rightarrow 0, \quad m \rightarrow \infty
$$

we also obtain that $\nabla u_{m}$ converges in measure to $\nabla u$ in $A$, and $H\left(\nabla u_{m}\right)$ converges in measure to $H(\nabla u)$.

We continue with the following Proposition.

Proposition 5.2. $u_{m} \in C^{\infty}(\Omega)$. Also, for any compact $K \Subset \Omega$ and $\delta>0$, $\nabla u_{m}(K)$ is in a $\delta$-neighborhood of $\bar{N}$ for $m$ large enough. 
Proof. The proof is standard and follows the lines of interior Lipschitz estimates for $p$-harmonic functions.

Using the lower obstacle $\varphi$ as competitor, we see that $\int_{\Omega} F_{m}\left(\nabla u_{m}\right) d x$ is bounded above by a fixed constant. Hence,

$$
\int_{\Omega} \bar{F}\left(\nabla u_{m}\right) d x \rightarrow 0, \text { as } m \rightarrow+\infty .
$$

For notational simplicity we denote $u_{m}=\tilde{u}$ and $F_{m}=\widetilde{F}$.

Let $\eta$ be a convex function, $\eta=0$ outside $D_{m_{0}}=\left\{\bar{F}>1 / m_{0}\right\}$ for some fixed $m_{0}, \eta(p)=|p|$ for large $|p|$. Also, let

$$
\psi=\eta(\nabla \tilde{u})+\frac{1}{2} \eta^{2}(\nabla \tilde{u}) .
$$

Then $\psi$ is a subsolution to the following elliptic equation

$$
\partial_{i}\left(a_{i j} \psi_{j}\right) \geq 0, \quad a_{i j}=\frac{\bar{F}_{i j}(\nabla \tilde{u})}{1+\eta(\nabla \tilde{u})} .
$$

Indeed, since $\widetilde{F}=C_{m} \bar{F}$ in $D_{m_{0}}$ (we assume $m>m_{0}$ ) and $\eta, \psi=0$ when $\nabla \tilde{u}$ is outside $D_{m_{0}}$, we can replace $\bar{F}$ by $\widetilde{F}$ in our computations. Using the Euler-Lagrange equation for $\tilde{u}$

$$
\partial_{i}\left(\widetilde{F}_{i j} \tilde{u}_{k j}\right)=0
$$

it is straightforward to check that

$$
\partial_{i}\left(a_{i j} \psi_{j}\right)=\widetilde{F}_{i j} \eta_{k l} \tilde{u}_{l i} \tilde{u}_{k j} \geq 0 .
$$

Notice that the equation (5.2) is uniformly elliptic since in $D_{m_{0}}$ we have

$$
\lambda_{m_{0}}(1+\eta) I \leq D^{2} \bar{F} \leq \Lambda_{m_{0}}(1+\eta) I .
$$

Now we can apply the standard estimate (see GT, Theorem 8.17) and obtain that for any compact $K \Subset \Omega$,

$$
\|\psi\|_{L^{\infty}(K)} \leq C\|\psi\|_{L^{\alpha}(\Omega)}, \quad \text { for } \alpha>1,
$$

with $C$ depending on $m_{0}, K, \alpha$. We choose $\alpha=3 / 2$ and use

$$
\psi^{3 / 2} \leq C\left(1+|\nabla \tilde{u}|^{3}\right) \leq C\left(m_{0}\right) \bar{F}(\nabla \tilde{u})
$$

together with (5.1) to obtain

$$
\|\psi\|_{L^{\infty}(K)} \rightarrow 0 \text { as } m \rightarrow \infty,
$$

which implies the second statement of our proposition.

Since $\nabla \tilde{u}$ is locally bounded the first part $\left(\tilde{u} \in C^{\infty}\right)$ follows from the classical theory.

Remark. The smoothness of $u_{m}$ follows also from the fact that $u_{m}$ solves a uniformly elliptic equation in $2 \mathrm{D}$, therefore $u_{m}$ is $C^{1, \alpha}$ in the interior and hence it is $C^{\infty}$.

Our analysis will rely on the following classical theorem that was also used in other two dimensional results (see for example $[\mathrm{GT},[\underline{\mathrm{H}},[\underline{\mathrm{S}}]$ ). 
Theorem 5.3. Let $v$ be a solution to

$$
a_{i j}(x) v_{i j}(x)=0 \quad \text { in } D \subset \mathbb{R}^{2},
$$

with $A(x)=a_{i j}(x)>0, A(x) \in C^{\infty}$, and $D$ simply connected. Assume $v$ is not linear. Then in each neighborhood $U$ there exists a point $x_{U}$ such that each set

$$
\left\{v>l_{U}\right\}, \quad\left\{v<l_{U}\right\}
$$

with

$$
l_{U}(x):=v\left(x_{U}\right)+\nabla v\left(x_{U}\right) \cdot\left(x-x_{U}\right)
$$

has at least two connected components in $D$ that intersect $U$. Moreover these components are not compactly supported in D.

Proof. Let $x_{U} \in U$ be such that $\operatorname{det} D^{2} v\left(x_{U}\right)<0$. Such a point exists otherwise if $\operatorname{det} D^{2} v=0$ in $U$, then $D^{2} v=0$ in $U$ and by unique continuation $v$ is linear in $D$.

Clearly, the sets $\left\{v>l_{U}\right\},\left\{v<l_{U}\right\}$ intersect a small ball around $x_{U}$ precisely in four disjoint connected components.

On the other hand, it follows from the maximum principle that any connected component of the sets $\left\{v>l_{U}\right\},\left\{v<l_{U}\right\}$ cannot be compactly supported in $D$. Hence, since $D \subset \mathbb{R}^{2}$ and $D$ is simply connected, the components in the small ball belong to four disjoint connected components in $D$.

\section{The PRoof of Theorem 1.3 .}

In this section we exhibit the proof of Theorem 1.3. We start by obtaining a result to which we refer to as the localization Theorem. From now on we tacitly assume that our statements hold for all $m$ sufficiently large.

Theorem 6.1. Assume $B_{1} \subset \Omega$ and

$$
\nabla u_{m}\left(B_{1}\right) \cap \mathcal{B}_{\rho}\left(p_{0}\right)=\emptyset, \quad p_{0} \in \bar{N} .
$$

Then for any $\varepsilon>0$, there exists $\delta=\delta\left(\varepsilon, F, \mathcal{B}_{\rho}\left(p_{0}\right)\right)$ such that either

$$
\nabla u_{m}\left(B_{\delta}\right) \subset \mathcal{B}_{\varepsilon}(p) \text {, for some } p \in N,
$$

or

$$
\nabla u_{m}\left(B_{\delta}\right) \subset \mathcal{N}_{\varepsilon}
$$

with $\mathcal{N}_{\varepsilon}$ the $\varepsilon$ neighborhood of $\partial N$.

Remark. Another way of stating the conclusion of this theorem is to say that $H\left(\nabla u_{m}\right)$ is continuous with a uniform modulus of continuity.

Also recall that $H\left(\nabla u_{m}\right) \rightarrow H(\nabla u)$ in measure, thus if the hypothesis of Theorem 6.1 holds for all large $m$ 's, then $u$ satisfies the same conclusion of the Theorem.

Proof. Assume

$$
\nabla u_{m}\left(B_{1}\right) \cap \mathcal{B}_{r}(p)=\emptyset, \quad \overline{\mathcal{B}_{4 r}(p)} \subset N \backslash \mathcal{Q} .
$$

We wish to prove that there exists $\delta>0$ depending on $F, \mathcal{B}_{r}(p)$, such that either

$$
\nabla u_{m}\left(B_{\delta}\right) \subset \mathcal{B}_{4 r}(p)
$$

or

$$
\nabla u_{m}\left(B_{\delta}\right) \cap \mathcal{B}_{3 r}(p)=\emptyset .
$$


We argue similarly as for Lemma 3.1. It suffices to prove the following Caccioppolitype inequality,

$$
\int_{B_{1 / 2} \cap\left(\nabla u_{m}\right)^{-1}\left(\mathcal{B}_{4 r}(p)\right)}\left|D^{2} u_{m}\right|^{2} d x \leq C,
$$

for some constant $C$ depending on $F, \mathcal{B}_{r}(p)$.

For notational simplicity let $F_{m}=\widetilde{F}$ and $u_{m}=\tilde{u}$. We have

$$
\int_{B_{1}} \widetilde{F}_{i j}(\nabla \tilde{u}) \tilde{u}_{k j} \phi_{i} d x=0, \forall \phi \in C_{0}^{\infty}\left(B_{1}\right) .
$$

Let us choose $\phi=\xi^{2} \eta_{k}(\nabla \tilde{u})$, with $\eta$ compactly supported on $\overline{\mathcal{B}_{4 r}(p)}$ and $\xi \in$ $C_{0}^{\infty}\left(B_{1}\right), 0 \leq \xi \leq 1, \xi \equiv 1$ on $B_{1 / 2}$. We obtain, after summing over all $k$ 's (when clear, we drop the dependence on $\nabla \tilde{u}$ ),

$$
\int_{B_{1}} \widetilde{F}_{i j} \tilde{u}_{k j} \xi^{2}\left(\eta_{k}(\nabla \tilde{u})\right)_{i} d x=-2 \int_{B_{1}} \widetilde{F}_{i j} u_{k j} \xi \xi_{i} \eta_{k} d x
$$

Now we analyze the left-hand side of (6.5). We proceed as in Lemma 3.2 and we obtain

$$
\begin{aligned}
L H S & =\int_{B_{1}} \widetilde{F}_{i j} \tilde{u}_{k j} \xi^{2} \eta_{k l} \tilde{u}_{l i} d x \\
& =\int_{B_{1}} \xi^{2}\left(\operatorname{det} D^{2} \widetilde{F}\right)\left|\operatorname{det} D^{2} \tilde{u}\right| \operatorname{Tr}\left(\left(D^{2} \widetilde{F}\right)^{-1} D^{2} \eta\right) d x .
\end{aligned}
$$

Choose $\eta$ such that

$$
\operatorname{Tr}\left(\left(D^{2} \widetilde{F}\right)^{-1} D^{2} \eta\right) \geq c, \quad \text { on } \mathcal{B}_{4 r}(p) \backslash \mathcal{B}_{r}(p),
$$

for a small constant $c$ depending on the ellipticity constants of $D^{2} F$ on $\mathcal{B}_{4 r}(p)$, say $\lambda, \Lambda$. We conclude

$$
L H S \geq c \int_{B_{1} \cap(\nabla \tilde{u})^{-1}\left(\mathcal{B}_{4 r}(p)\right)}\left|D^{2} \tilde{u}\right|^{2} \xi^{2} d x
$$

with $c$ depending on $\lambda, \Lambda$.

On the other hand, the right-hand side of (6.5) is bounded by

$$
|R H S| \leq \gamma C \int_{B_{1} \cap(\nabla \tilde{u})^{-1}\left(B_{4 r}(p)\right)}\left|D^{2} \tilde{u}\right|^{2} \xi^{2} d x+\tilde{C}
$$

with $C$ depending on $\Lambda$. Combining (6.5), (6.6) and (6.7) we obtain the desired inequality.

We now proceed similarly as in the proof of Theorem 1.2. We cover the set $N \backslash\left(\mathcal{N}_{\varepsilon} \cup \mathcal{B}_{\varepsilon}\left(q_{i}\right)\right)$ as a finite union of balls $\mathcal{B}_{r}^{k}$ of radius $r=\varepsilon / 5$ (with centers in the set). From our assumption, there exists $k$ such that

$$
\nabla u_{m}\left(B_{1}\right) \cap \mathcal{B}_{r}^{k}=\emptyset,
$$

provided that $\varepsilon$ is small enough depending on $\rho, F$. Now the conclusion follows from the same iteration argument as in Theorem 1.2 (notice that by Proposition 5.2. $\nabla u_{m}\left(B_{\delta}\right)$ is in an $\varepsilon$ neighborhood of $\bar{N}$ for large $m$.) 
6.1. The proof of Theorem 1.3. We now present a series of Propositions which will all be combined towards the proof of Theorem 1.3. We start by stating two Propositions for the approximation $u_{m}$ which correspond to the flatness Theorems 4.34 .4 for the minimizer $u$. We present their proofs in the last section.

Proposition 6.2. Assume $B_{1} \subset \Omega$ and

$$
\mathcal{B}_{\delta}\left(p_{0}\right) \subset N
$$

Then, there exists $\varepsilon$ depending on $\delta, p_{0}, F$, such that if

$$
\left|u_{m}-p_{0} \cdot x\right| \leq \varepsilon, \quad x \in B_{1}
$$

then

$$
\nabla u_{m}\left(B_{1 / 2}\right) \subset \mathcal{B}_{\delta}\left(p_{0}\right) .
$$

Proposition 6.3. Let $p_{0} \in \partial N$. Then, there exists $\varepsilon$ depending on $\delta, F$, such that if

$$
\left|u_{m}-p_{0} \cdot x\right| \leq \varepsilon, \quad x \in B_{1}
$$

then

$$
\left[\nabla u_{m}(x), p_{0}\right] \quad \text { is in a } \delta \text {-neighborhood of } \partial N \text {, for all } x \in B_{1 / 2} \text {. }
$$

In order to apply the localization Theorem near the origin (see (6.1)), we need to find a ball $\mathcal{B}_{r}(p), p \in \bar{N}$, that does not intersect the image $\nabla u_{m}\left(B_{r}\right)$ (with $r$ small and $\mathcal{B}_{r}(p)$ depending also on $u$ ). This is not always possible.

The next Proposition, which is key in proving Theorem 1.3, states a condition which guarantees the existence of such a ball in a neighborhood of a side of $N$. Its proof relies on the previous flatness results.

Proposition 6.4. Assume $0 \in\left(p_{1}, p_{2}\right)$ and $e_{2}$ is normal to $\left(p_{1}, p_{2}\right)$ and points inside $N$. Let $0 \in\left[a_{1}, a_{2}\right] \subset\left(p_{1}, p_{2}\right)$ and assume that for each $r>0$ and $p \in\left[a_{1}, a_{2}\right]$, there exists a sequence of $m \rightarrow \infty$ such that

$$
\nabla u_{m}\left(B_{r}\right) \cap \mathcal{B}_{r}(p) \neq \emptyset .
$$

Then $u$ is constant on a segment of direction $e_{2}$ connecting 0 and $\partial \Omega$. Precisely, there exists $\tilde{x}=s e_{2} \in \partial \Omega$ such that

$$
u(x)=u(0), \quad \text { for all } x \in[0, \tilde{x}] \subset \bar{\Omega} .
$$

In order to prove Proposition 6.4 we will need the following Lemma.

Lemma 6.5. Assume $0 \in \partial N$, $e_{2}$ points inside $N$,

$$
R:=\left\{\left|x_{1}\right|<\delta,\left|x_{2}\right|<1\right\} \Subset \Omega,
$$

and

$$
u(x)>0, x \in \bar{R} \cap\left\{x_{2}=1\right\}, \quad u(x)<0, x \in \bar{R} \cap\left\{x_{2}=-1\right\}, \quad u(0)=0 .
$$

Assume that for each $r>0$, there exists a sequence of $m \rightarrow \infty$ such that

$$
\nabla u_{m}\left(B_{r}\right) \cap \mathcal{B}_{r}(0) \neq \emptyset \text {. }
$$

Then either the set

or the set

$$
\{u=0\} \cap\left\{x_{1}>0\right\}
$$

$$
\{u=0\} \cap\left\{x_{1}<0\right\}
$$

is given by the region between a convex graph (from above) and a concave graph (from below) in the $e_{2}$ direction. 
Proof. The lemma holds trivially if $u$ is linear. Assume $u$ is not linear. Then, for $m$ large enough, also $u_{m}$ is not linear. Then, by the assumptions together with Theorem [5.3] we have that there exists $x_{m} \rightarrow 0$ with $\nabla u_{m}\left(x_{m}\right) \rightarrow 0$ such that the set

$$
\left\{u_{m}<l_{m}:=u_{m}\left(x_{m}\right)+\nabla u_{m}\left(x_{m}\right) \cdot\left(x-x_{m}\right)\right\}
$$

has at least two distinct components in $\bar{R}$. One of the components that does not contain the segment $\bar{R} \cap\left\{x_{2}=-1\right\}$ must intersect one of the lateral sides, say $x_{1}=\delta$ (for infinitely many $m$ 's.) Then we can find a polygonal line connecting any neighborhood of $x_{m}$ with $x_{1}=\delta$ that is included in this component.

Now, for each $t \in(0, \delta]$ we define $\underline{h}(t), \bar{h}(t)$ to be the points in $\mathbb{R}^{2}$ on the line $x_{1}=t$ such that

$$
[\underline{h}(t), \bar{h}(t)]=\{u=0\} \cap\left\{x_{1}=t\right\},
$$

(notice that $u$ is increasing in the $e_{2}$ direction.) Let $c \in(a, b) \subset(0, \delta]$.

Claim. The segment $[\bar{h}(a), \bar{h}(b)]$ is above $\underline{h}(c)$ in the $e_{2}$ direction.

Indeed, assume by contradiction that our claim does not hold. Then, there exists a linear function $l$ increasing in the $e_{2}$ direction, such that

$$
[\bar{h}(a), \bar{h}(b)] \subset\{l<-1\}, \quad \underline{h}(c) \in\{l>1\} .
$$

Let $R_{a b}:=\left\{a<t<b,\left|x_{2}\right|<1\right\}$. Denote by $U_{m}$ the connected component of $\left\{u_{m}<l_{m}\right\}$ in $\overline{R_{a b}}$ that contains the segment $\overline{R_{a b}} \cap\left\{x_{2}=-1\right\}$. We compare $u_{m}$ and $l_{m}+\varepsilon l$ in the set $\overline{R_{a b}} \backslash U_{m}$, with $\varepsilon$ small enough depending on $u$ and $l$. We have

$$
u-\varepsilon l>0 \text { on } \overline{R_{a b}} \cap\left\{x_{2}=1\right\}
$$

and

$$
u-\varepsilon l>0 \text { on }\{u \geq-\varepsilon / 2\} \cap\left(\left\{x_{1}=a\right\} \cup\left\{x_{1}=b\right\}\right) .
$$

Notice that, since $u_{m} \rightarrow u, l_{m} \rightarrow 0$ uniformly, we have that

$$
\{u<-\varepsilon / 2\} \cap \overline{R_{a b}} \subset U_{m}
$$

for all $m$ large enough, thus

$$
u_{m}-l_{m}-\varepsilon l>0 \quad \text { on } \partial R_{a b} \backslash U_{m} .
$$

On the other hand, $u$ is strictly negative on the segment $\left\{x_{1}=c\right\} \cap\{l<0\} \cap \overline{R_{a b}}$. Thus this segment is included in $U_{m}$, which implies that

$$
u_{m}-l_{m}-\varepsilon l<0 \quad \text { on } \partial U \cap\left\{x_{1}=c\right\} \cap R_{a b} .
$$

Hence the minimum of $u_{m}-l_{m}-\varepsilon l$ in $\overline{R_{a b}} \backslash U_{m}$ is negative and by the maximum principle it occurs at some point $x_{0} \in \partial U_{m} \cap R_{a b}$. Thus,

$$
u_{m}-l_{m} \geq \varepsilon\left(l-l\left(x_{0}\right)\right) \quad \text { in } \overline{R_{a b}} \backslash U_{m} .
$$

Recall that there exists a polygonal line included in $\left(\overline{R_{a b}} \backslash U_{m}\right) \cap\left\{u_{m}<l_{m}\right\}$ that connects the lines $x_{1}=a$ and $x_{2}=b$. Now the right-hand side of (6.8) is increasing in the $e_{2}$ direction and we obtain a contradiction at a point where $x_{0}+s e_{2}, s \geq 0$ intersects this polygonal line. Thus the claim is proved. 
Next we prove that $\bar{h}(t)$ is a convex curve and $\underline{h}(t)$ is a concave curve, if $t>0$. Indeed, let $0<t_{1}<t_{2} \leq \delta$, and let $Q$ be the convex set generated by $\bar{h}\left(t_{i}\right), \underline{h}\left(t_{i}\right), i=$ 1,2 . From the claim above we see that

$$
Q \cap[\underline{h}(t), \bar{h}(t)] \neq \emptyset, \quad t_{1} \leq t \leq t_{2}
$$

Thus,

$$
u \leq 0 \text { on }\left[\underline{h}\left(t_{1}\right), \underline{h}\left(t_{2}\right)\right] .
$$

Let $l^{\prime}$ be the linear function that is 0 on the line passing through $\underline{h}\left(t_{i}\right)-\gamma e_{2}, i=1,2$ and has slope $\varepsilon$ in the $e_{2}$ direction. Clearly, if $\varepsilon$ is small depending on $u$ and $\gamma$, then $u \leq l^{\prime}$ on the boundary of $\left\{t_{1}<x_{1}<t_{2}, x_{2} \geq-1\right\} \cap\left\{l^{\prime} \leq 0\right\}$. Hence the same inequality is true in the interior. Thus

$$
u<0, \quad \text { below }\left[\underline{h}\left(t_{1}\right), \underline{h}\left(t_{2}\right)\right]-\gamma e_{2} .
$$

By letting $\gamma$ tend to 0 and repeating the same argument from above, we find that $[\underline{h}(t), \bar{h}(t)] \subset Q$.

Let $\left[a_{1}, a_{2}\right] \subset\left(p_{1}, p_{2}\right)$ with $e_{2}$ normal to $\left(p_{1}, p_{2}\right)$ and pointing inside $N$. For all $p \in\left[a_{1}, a_{2}\right]$ with the property that for each $r>0$ there exists a sequence of $m \rightarrow \infty$ such that

we define

$$
\nabla u_{m}\left(B_{r}\right) \cap \mathcal{B}_{r}(p) \neq \emptyset
$$

$$
C_{p}:=\{u=p \cdot x\} .
$$

Assume that $R:=\left\{\left|x_{1}\right|<\delta,\left|x_{2}\right|<1\right\} \subset \Omega$ with

$$
u>0 \text { on } \bar{R} \cap\left\{x_{2}=1\right\}, \quad u<0, \text { on } \bar{R} \cap\left\{x_{2}=-1\right\}, \quad u(0)=0 .
$$

Then for each $p \in\left[a_{1}, a_{2}\right]$, possibly by taking $\delta$ smaller, we also have

$$
u(x)>p \cdot x \text {, on } \bar{R} \cap\left\{x_{2}=1\right\}, \quad u(x)<p \cdot x \text {, on } \bar{R} \cap\left\{x_{2}=-1\right\} .
$$

Thus applying Lemma 6.5 (with the origin replaced by $p$ ) for each slope $p$ as above, either the set

$$
C_{p}^{+}:=C_{p} \cap\left\{0<x_{1}<\delta\right\}
$$

or the set

$$
C_{p}^{-}:=C_{p} \cap\left\{0<x_{1}<\delta\right\}
$$

is given by the region between a convex function and a concave function. Let $A^{+}$ (resp. $A^{-}$) be the set of $p \in\left[a_{1}, a_{2}\right]$ such $C_{p}^{+}$(resp. $C_{p}^{-}$) is given by such region.

With this notation, we state and prove the following Lemma which will be used for the proof of Proposition 6.4

Lemma 6.6. Let $p \in A^{+}$and $\alpha \in(0, \delta)$. Then, for any neighborhoods $V$ of $C_{p}^{+} \cap\left\{x_{1}=\alpha\right\}$ and $W$ of $p$, there exist a point $\tilde{x} \in V$ such that $\nabla u_{m}(\tilde{x}) \in W$.

Proof. Without loss of generality we assume $p=0 \in A^{+}$. We refer to the proof of the previous lemma. We can assume that $u_{m}$ is not linear, otherwise the statement is trivial. Let $\alpha \in(a, b) \subset(0, \delta)$, and let us focus on the connected component $\widetilde{U}_{m}$ of $\left\{u_{m}<l_{m}\right\}$ which contains the polygonal line connecting $x_{1}=a$ with $x_{1}=b$. Since $u_{m} \rightarrow u, l_{m} \rightarrow 0$ uniformly, we obtain that $\widetilde{U}_{m}$ is in any neighborhood of $C_{p}$, if $m$ is large enough. Now, consider the function $u_{m}-l_{m}+\frac{1}{2}\left(x_{1}-\alpha\right)^{2}$. Then the 
minimum of this function in $\widetilde{U}_{m}$ is negative and is achieved at an interior point $\tilde{x}$. The desired conclusion follows by taking the interval $(a, b)$ sufficiently small.

We are now ready to exhibit the proof of Proposition 6.4

Proof of Proposition 6.4. We take $u(0)=0$ and use the notation of the previous Lemmas. Assume by contradiction that $\{u=0\}$ does not contain either of the segments in the direction $e_{2}$ connecting 0 with $\partial \Omega$. Then there exists $\delta$ small such that $R \Subset \Omega, u>0$ on $\bar{R} \cap\left\{x_{2}=1\right\}$ and $u<0$ on $\bar{R} \cap\left\{x_{2}=-1\right\}$. Therefore, given $\left[a_{1}, a_{2}\right] \subset\left(p_{1}, p_{2}\right)$, we can define $A^{+}$and $A^{-}$as above (see discussion before Lemma 6.6.)

Since $\overline{A^{+}} \cup \overline{A^{-}}=\left[a_{1}, a_{2}\right]$, then there exists an open interval contained in either $\overline{A^{+}}$or $\overline{A^{-}}$, say $\left(-\rho e_{1}, \rho e_{1}\right) \subset \overline{A^{+}}$.

Now set

$$
D_{t}:=\left\{u=t e_{1} \cdot x\right\} \cap\left\{0<x_{1}<\delta\right\}, \quad-\rho<t<\rho .
$$

Notice that if $t e_{1} \in A^{+}$then $D_{t}$ is exactly $C_{t e_{1}}^{+}$.

We claim that $D_{t}$ is precisely the region between two segments with one endpoint on $\left\{x_{1}=0\right\}$ and the other on $\left\{x_{1}=\delta\right\}$.

Indeed, let $t_{n} e_{1} \in A^{+}, t_{n} \uparrow t \in(-\rho, \rho), t_{n}$ strictly increasing. Notice that, if $x_{1}>0$

$$
\left\{u<t x_{1}\right\}=\bigcup_{n}\left\{u<t_{n} x_{1}\right\}=\bigcup_{n}\left\{u \leq t_{n} x_{1}\right\} .
$$

Using that $C_{t_{n} e_{1}}^{+}$is the region between a convex and a concave graph, we obtain from (6.10) that $\partial\left\{u<t x_{1}\right\} \cap\left\{0<x_{1}<\delta\right\}$ is both a concave and a convex graph. This implies the claim.

Next, choose a point of differentiability $x^{*}$ for $u$ in the open set

$$
\left\{-\rho e_{1} \cdot x<u<\rho e_{1} \cdot x\right\} \cap\left\{0<x_{1}<\delta\right\},
$$

such that

$$
\nabla u\left(x^{*}\right) \cdot e_{2}>\gamma>0 .
$$

Set $p^{*}=\nabla u\left(x^{*}\right)$. Without loss of generality we can assume $u\left(x^{*}\right)=0$ and thus the set $D_{0}$ consists of just one segment. Therefore, if $t_{n} \uparrow 0, t_{n}$ increasing, $t_{n} e_{1} \in A^{+}$, then

$$
C_{t_{n} e_{1}}^{+} \cap\left\{e_{1} \cdot\left(x-x^{*}\right)=0\right\} \rightarrow x^{*} .
$$

Since $x^{*}$ is a point of differentiability of $u$ and $u_{m} \rightarrow u$ uniformly, we obtain that for any $\varepsilon>0$ we can find $\rho, m$ such that

$$
\left|u_{m}-p^{*} \cdot\left(x-x^{*}\right)\right| \leq \varepsilon \rho \quad \text { in } B_{\rho}\left(x^{*}\right) .
$$

On the other hand from (6.12) together with Lemma 6.6, we can find a point $x_{m} \in B_{\rho / 2}$ such that

$$
\left|\nabla u_{m}\left(x_{m}\right)\right| \leq \gamma / 2
$$

Then, using (6.11) we see that the middle point of $\left[\nabla u_{m}\left(x_{m}\right), p^{*}\right]$ is outside a $\gamma / 4$ neighborhood of $\partial N \cup\left\{p^{*}\right\}$ (provided that $\gamma$ is chosen small enough depending on $N$ ). This contradicts either Proposition 6.2 (if $p^{*} \in N$ ) or Proposition 6.3 (if $\left.p^{*} \in \partial N\right)$, by choosing $\varepsilon=\varepsilon\left(\gamma, p^{*}, N\right)$ sufficiently small. 
Now, we are finally ready to present the proof of Theorem 1.3.

Proof of Theorem 1.3. For simplicity take $x_{0}=0$. Assume that there are no segments connecting 0 with $\partial \Omega$ as in the statement of the Theorem. We wish to prove that given $\rho>0$ there exists $\delta$ (depending also on $u$ ) small such that $\operatorname{diam}\left(\nabla u\left(B_{\delta}\right)\right) \leq \rho$.

By Proposition 6.4 for each side $\left[p_{i}, p_{i+1}\right]$ and each subinterval $\left(a_{i}, a_{i+1}\right) \subset$ $\left[p_{i}, p_{i+1}\right]$ there exist a point $a \in\left(a_{i}, a_{i+1}\right)$ and $r>0$ such that

$$
\nabla u_{m}\left(B_{r}\right) \cap \mathcal{B}_{r}(a)=\emptyset .
$$

Clearly we can find $\varepsilon>0$ and points $a_{k} \in \partial N$ such that any ball $\mathcal{B}_{\rho / 2}(p)$ centered at $p \in \partial N$ contains one of the balls $\mathcal{B}_{\varepsilon}\left(a_{k}\right)$ and

$$
\nabla u_{m}\left(B_{\varepsilon}\right) \cap \mathcal{B}_{\varepsilon}\left(a_{k}\right)=\emptyset .
$$

We cover the open set

$$
O=\{x \in N \mid \operatorname{dist}(x, \partial N)>\varepsilon / 2\}
$$

with a finite number of balls of radius $\varepsilon / 10$ centered in the set. According to the localization Theorem 6.1 if $\delta$ is small enough depending only on $F, \varepsilon$ and say $\mathcal{B}_{\varepsilon}\left(a_{1}\right)$ we find that either

$$
\operatorname{diam}\left(\nabla u_{m}\left(B_{\delta}\right)\right) \leq \varepsilon / 5
$$

or

$$
\nabla u_{m}\left(B_{\delta}\right) \cap O=\emptyset .
$$

In the latter case we know from Proposition 5.2 that for $m$ large enough

$$
\nabla u_{m}\left(B_{\delta}\right) \text { is included in a } \varepsilon / 2 \text {-neighborhood of } N \text {. }
$$

Since $\nabla u_{m}\left(B_{\delta}\right)$ is connected, we conclude from the choice of $\varepsilon$, (6.14) and (6.15) that

$$
\operatorname{diam}\left(\nabla u_{m}\left(B_{\delta}\right)\right) \leq \rho .
$$

The conclusion follows by letting $m \rightarrow \infty$.

We conclude this Section with the proof of Theorem 4.2

Proof of Theorem 4.2. For simplicity assume $p_{i}=0, c=0$ and $\omega=e_{2}$. Since $u$ is increasing in a cone of directions around $e_{2}$ we conclude that $S$ is between two Lipschitz graphs in the $e_{2}$ direction. Assume by contradiction that the lower graph is not concave. Then we can find two points $z_{1}, z_{2}$ such that $u\left(z_{i}\right)<0$ and the segment $\left[z_{1}, z_{2}\right]$ is tangent from below to $S$. Let $l$ be the linear function which vanishes at the points $z_{i}+\varepsilon e_{2}$ with $\nabla l \cdot e_{2}=\varepsilon$. We compare $u$ and $l$ in the set $\left\{x \cdot z_{1}<x_{1}<x \cdot z_{2}\right\} \cap\{l<0\} \cap \Omega$. If $\varepsilon$ is small enough then $u \leq l$ on the boundary, hence $u \leq l$ in this set. This implies that $S$ is above the segment $\left[z_{1}, z_{2}\right]+\varepsilon e_{2}$ and we contradict the fact that $\left[z_{1}, z_{2}\right]$ was tangent to $S$. 


\section{The proof of Theorem 4.1}

We distinguish two cases, when the polygon $N$ has $n \geq 4$ vertices and when it has only $n=3$ vertices. The latter is more involved and we only present a sketch of the proof.

Lemma 7.1. Assume $N$ has more that 3 vertices. Then $H(\nabla u)$ is continuous.

Proof. If $H(\nabla u)$ is not continuous at the origin, then for each side $\left[p_{i}, p_{i+1}\right]$ there exists a segment of direction $\nu_{i}$ perpendicular to that side, starting from the origin and ending at $x_{i} \in \partial \Omega$ such that $u(x)=u(0)+p_{i} \cdot x, x \in\left[0, x_{i}\right]$. This follows from the localization Theorem and from Proposition 6.4.

For simplicity of exposition, assume that

$$
p_{1}=\left|p_{1}\right|(-\cos \theta, \sin \theta), \quad p_{2}=0, \quad p_{3}=\left|p_{3}\right|(\cos \theta, \sin \theta), \quad \theta \in(0, \pi / 2) .
$$

Recall that in our notation $\nu_{i}$ is either of the two directions perpendicular to $\left[p_{i}, p_{i+1}\right]$. Let us choose $\nu_{1}, \nu_{2}$ pointing inside $N$, that is

$$
\nu_{1}=(\sin \theta, \cos \theta), \quad \nu_{2}=(-\sin \theta, \cos \theta) .
$$

Assume $u(0)=0$, then $u=0$ on two segments starting at 0 of directions $\pm \nu_{1}$ and $\pm \nu_{2}$ respectively. Denote by $\vartheta_{2}$ the closed angle (smaller than $\pi$ ) generated by these two directions. We wish to prove that

$$
\lim _{x \rightarrow 0, x \in \vartheta_{2}} H(\nabla u)=H(0),
$$

and moreover that no segment of direction $\nu_{j}, j \neq 1,2$ along which $u=p_{j} \cdot x$ can intersect the angle $\vartheta_{2}$.

We distinguish two cases.

Case 1. $u=0$ along segments of directions $\nu_{i}, i=1,2$ (or analogously $-\nu_{i}$, $i=1,2$.)

Since $\nabla u \in \bar{N}$, then $u$ is increasing in the cone of directions in $\vartheta_{2}$, thus $u \geq 0$ in $\vartheta_{2}$. We also have that $u-p_{1} \cdot x$ is increasing in the direction $(\sin (\theta+\delta), \cos (\theta+\delta))$ with $\delta>0$ small depending on $N$. This implies that near the origin in $\vartheta_{2}$ we have that $u \leq p_{1} \cdot x$, and analogously $u \leq p_{3} \cdot x$. Thus, in $B_{\rho} \cap \vartheta_{2}$ we have

$$
0 \leq u \leq \min \left\{p_{1} \cdot x, p_{3} \cdot x\right\}
$$

We use the hypothesis $n \geq 4$ to prove that no segment of direction $\nu_{j}, j \neq 1,2$ in which $u=p_{j} \cdot x$ can intersect the angle $\vartheta_{2}$. Indeed, in this angle near the origin we have $u \leq p \cdot x$ for all $p \in\left[p_{1}, p_{3}\right]$ and also $p \cdot x>0$ when $p \in\left(p_{1}, p_{3}\right)$. If $u=p_{j} \cdot x$ on a segment in the interior of the angle $\vartheta_{2}$ then we reach a contradiction since there exists $0<\lambda<1$ such that $\lambda p_{j} \in\left(p_{1}, p_{3}\right)$ and on this segment $u>\lambda p_{j} \cdot x \geq u$.

In $B_{\rho}$ we consider the function $\tilde{u}$ such that $\tilde{u}=u$ in the angle $\vartheta_{2}$ and $\tilde{u}=0$ outside this angle. Then, $\tilde{u}$ is a minimizer for $I$ in $B_{\rho}$. This follows from the fact that the gradient of the minimizer with boundary data $\tilde{u}$ on $\partial B_{\rho}$ belongs to $\bar{N}$ and therefore the minimizer must be bounded by 0 from below and by $\max \left\{p_{i} \cdot x, 0\right\}, i=$ 1,3 from above.

Clearly if $j \geq 3$, then $p_{j} \cdot \nu_{j} \neq 0$ while $\tilde{u}=0$ outside the angle $\vartheta_{2}$. This implies that $\tilde{u} \neq p_{j} \cdot x$, on a segment of direction $\nu_{j}$. Thus we conclude from the localization 
Theorem and from Proposition 6.4 that $H(\nabla \tilde{u})$ is continuous and

$$
\lim _{x \rightarrow 0, x \in \vartheta_{2}} H(\nabla u)=\lim _{x \rightarrow 0} H(\nabla \tilde{u})=H(0) .
$$

Case 2. $u=0$ along segments of directions $\nu_{1}$, and $-\nu_{2}$ (or analogously $-\nu_{1}, \nu_{2}$.)

Then $u=0$ in $B_{\rho} \cap \vartheta_{2}$ and (7.1) clearly holds. Also, as in the conclusion of the previous case no segment of direction $\nu_{j}, j \geq 3$ in which $u=p_{j} \cdot x$ can intersect $\vartheta_{2}$.

Since the angles generated by consecutive directions cannot overlap, they must cover a neighborhood of the origin and the lemma is proved.

Lemma 7.2. If $N$ has three sides, $H(\nabla u)$ is continuous.

Sketch of the proof. We use the same notation as in the previous proof. The only case that does not follow from a similar analysis as before, is when $u$ is linear on segments of directions $\nu_{1}, \nu_{2}$ and $-\nu_{3}\left(\nu_{i}\right.$ points inside $\left.N\right)$. More precisely, $u=\min \left\{p_{1} \cdot x, p_{3} \cdot x\right\}$ in $B_{1} \cap \vartheta_{2}$, and $u \leq 0$ outside $\vartheta_{2}$. We can also assume that $u$ is not linear in the direction of $\nu_{3}$. We can assume further that $u$ is not linear on the segments of direction $-\nu_{1}$ (or $\left.-\nu_{2}\right)$. Otherwise, as in the proof of the previous Lemma, we can construct another minimizer $\tilde{u}=\min \left\{u, p_{1} \cdot x\right\}$ which coincides with $u$ on $\left\{p_{1} \cdot x \leq 0\right\}$. The continuity of $H(\nabla \tilde{u})$ (and hence of $H(\nabla u)$ ) follows as in the case $n \geq 4$.

In the remaining case we wish to prove that the assumption (6.1) of the localization Theorem holds, hence the Lemma follows.

First, suppose that there exist a direction $p \in\left(p_{1}, p_{3}\right)$ and a rectangle

$$
R_{a b}=\left\{a<x_{1}<b,\left|x_{2}\right|<1 / 2\right\}, \quad 0 \in(a, b) \subset[-1 / 2,1 / 2]
$$

such that

$$
u<0 \text { on } \partial R_{a b} \cap\{p \cdot x \leq 0\} .
$$

Then, for small $\varepsilon$ (depending on $u$ ) any linear function $l$ with

$$
\nabla l \in \mathcal{B}_{\varepsilon^{2}}(\varepsilon p), \quad|l(0)| \leq \varepsilon^{2}
$$

has the property that $\{u<l\} \cap \partial R_{a b}$ consists of one connected component. This implies that if we approximate $u$ in $R_{a b}$ by functions $u_{m}\left(u_{m}=u\right.$ on $\left.\partial R_{a b}\right)$, then

$$
\nabla u_{m}\left(B_{\varepsilon^{3}}\right) \cap \mathcal{B}_{\varepsilon^{2}}(\varepsilon p)=\emptyset,
$$

otherwise we contradict Theorem [5.3. Hence the Lemma follows from the localization Theorem.

The only case when we cannot find one pair $p,(a, b)$ satisfying (7.2)-(7.3), is when $u=0$ in $B_{\rho}$ ( $\rho$ small depending on $u$ ) above a line passing through the origin and below the angle $\vartheta_{2}$ (recall that $u$ is increasing in the $e_{2}$ direction). After a dilation, assume $\rho=1$. Therefore, we can assume that

$$
0 \geq u \geq \tilde{p} \cdot x, \quad \text { in } B^{-}:=B_{1} \cap\{\tilde{p} \cdot x \leq 0\},
$$

for some $\tilde{p} \in\left(p_{1}, p_{3}\right)$.

Now one can obtain a localization Theorem in $B^{-}$around the origin, even tough 0 is a boundary point. Indeed, let $u_{m}$ be the approximation for $u$ in $B^{-}\left(u_{m}=u\right.$ on $\left.\partial B^{-}\right)$. Then $\nabla u_{m}(x) \in[0, \tilde{p}]$ for all $x \in \partial B^{-} \cap\{\tilde{p} \cdot x=0\}$. Thus, we can obtain a Caccioppoli-type inequality at the boundary as in the proof of the localization 
Theorem, as long as the function $\eta$ in formula (6.5) (the domain of integration is now $\left.B^{-}\right)$is 0 on the segment $[0, \tilde{p}]$ and $\xi \in C_{0}^{\infty}\left(B_{1}\right)$. This implies that the iteration argument in the proof of the localization Theorem is valid provided that $\mathcal{B}_{4 r}^{k} \cap[0, \tilde{p}]=\emptyset$.

Also, a boundary version of Proposition 6.4 holds because the "thin" connected components of $\left\{u_{m}<l_{m}\right\}$ cannot intersect $\tilde{p} \cdot x=0$. More precisely, given $a_{i} \in$ $\partial N \backslash\{\tilde{p}\}$, one can show that there exists $r$ depending on $u$ and $a_{i}$ such that

$$
\nabla u_{m}\left(B_{r} \cap B^{-}\right) \cap \mathcal{B}_{r}\left(a_{i}\right)=\emptyset .
$$

Thus for any $\delta$, there exists $\varepsilon$ depending on $\delta$ and $u$ such that $\nabla u_{m}\left(B_{\varepsilon} \cap B^{-}\right)$ is in a $\delta$ neighborhood of the segment $[0, \tilde{p}]$. Letting $m \rightarrow \infty$ we obtain the same result for $u$.

Consider now a sequence of blow-up minimizers

$$
\frac{1}{r_{k}} u\left(r_{k} x\right), x \in B_{1},
$$

that converges to $\bar{u}$. Clearly, $\bar{u}$ is still a minimizer for $I$ and by the conclusion above $\nabla \bar{u}\left(B^{-}\right) \subset[0, \tilde{p}]$. This implies that $\bar{u}(x)=p \cdot x$ in $B^{-}$with $p \in[0, \tilde{p}]$. If $p \neq 0$, then we reach a contradiction since $\bar{u}$ is not a minimizer in a neighborhood of points $x$ such that $\tilde{p} \cdot x=0, x \neq 0$.

In conclusion for any $\varepsilon>0$ there exists $r_{\varepsilon}$ depending on $u$ and $\varepsilon$ such that

$$
\left|\frac{1}{r} u(r x)\right| \leq \varepsilon, \quad x \in B^{-}
$$

for all $r \leq r_{\varepsilon}$. Now the result follows applying the flatness Theorem 4.4 in balls $B_{c|x|}(x)$, for $c$ small enough and $x \in B^{-}$.

\section{The Proof of the flatness Theorems}

We finally present the proofs of Theorem 4.3 and Theorem 4.4. Since $u_{m} \rightarrow u$ uniformly, it suffices to prove Proposition 6.2 and Proposition 6.3.

Proposition 6.2. Assume $B_{1} \subset \Omega$ and

$$
\mathcal{B}_{\delta}\left(p_{0}\right) \subset N .
$$

Then, there exists $\varepsilon$ depending on $\delta, p_{0}, F$, such that if

$$
\left|u_{m}-p_{0} \cdot x\right| \leq \varepsilon, \quad x \in B_{1}
$$

then

$$
\nabla u_{m}\left(B_{1 / 2}\right) \subset \mathcal{B}_{\delta}\left(p_{0}\right)
$$

Proof. We can assume that $u_{m}$ is not linear, otherwise the result is obvious. Also, it suffices to show that the conclusion of our statement holds at 0 , i.e.

$$
\nabla u_{m}(0) \in \mathcal{B}_{\delta}\left(p_{0}\right), \quad \text { for large } m .
$$

First notice that

$$
\nabla u_{m}\left(B_{2 \sqrt{\varepsilon}}(0)\right) \cap \mathcal{B}_{2 \sqrt{\varepsilon}}\left(p_{0}\right) \neq \emptyset
$$

by considering $\min \left\{\frac{1}{2}|x|^{2}+u_{m}-p_{0} \cdot x\right\}$. 
Let $\mathcal{B}_{2 \rho}\left(p_{1}\right) \subset N$ such that $F \in C^{2}\left(\overline{\mathcal{B}_{2 \rho}\left(p_{1}\right)}\right), p_{0}$ not in $\mathcal{B}_{2 \rho}\left(p_{1}\right)$. We claim that

$$
\nabla u_{m}\left(B_{1 / 2}\right) \cap \mathcal{B}_{\rho}\left(p_{1}\right)=\emptyset, \quad \text { for small } \varepsilon \text {. }
$$

Clearly, the proposition follows from the claim, together with (8.1) and the localization Theorem. We are left with the proof of the claim.

Assume by contradiction that there exists $x_{0} \in B_{1 / 2}$ such that

$$
p_{2}:=\nabla u_{m}\left(x_{0}\right) \in \mathcal{B}_{\rho}\left(p_{1}\right) .
$$

We know that the set

$$
\left\{u_{m}(x)<l_{m}:=u_{m}\left(x_{0}\right)+p_{2} \cdot\left(x-x_{0}\right)\right\}
$$

has in $B_{1}$ at least two distinct connected components that intersect any neighborhood of $x_{0}$.

Notice that by the flatness assumption

$$
\left\{\left(x-x_{0}\right) \cdot\left(p_{0}-p_{2}\right)<-2 \varepsilon\right\} \subset\left\{u_{m}<l_{m}\right\}
$$

and

$$
\left\{\left(x-x_{0}\right) \cdot\left(p_{0}-p_{2}\right)>2 \varepsilon\right\} \subset\left\{u_{m}>l_{m}\right\} .
$$

This implies that one of the connected components of $\left\{u_{m}<l_{m}\right\}$ is included in the strip $\left\{\left|\left(x-x_{0}\right) \cdot\left(p_{0}-p_{2}\right)\right| \leq 2 \varepsilon\right\}$.

By changing the system of coordinates in the $x$ and $p$ spaces, we can assume that we have the following situation:

$$
\begin{gathered}
p_{2}=0, \quad p_{0}=\alpha e_{2}, \quad x_{0}= \pm e_{1} / 4, \quad u_{m}\left(x_{0}\right)=0 \\
\left|u_{m}-\alpha e_{2} \cdot x\right| \leq 2 \varepsilon \quad \text { in } \bar{R}:=\left\{\left|x_{1}\right| \leq 1 / 8,\left|x_{2}\right| \leq 1 / 8\right\}, \alpha>\rho .
\end{gathered}
$$

Moreover, the set $\left\{u_{m}<0\right\}$ has one connected component in $\bar{R}$ included in the strip $\left\{\left|x \cdot \alpha e_{2}\right| \leq 2 \varepsilon\right\}$ that intersects both $\left\{x_{1}= \pm 1 / 8\right\}$.

Let $U$ be the connected component that contains $\bar{R} \cap\left\{x_{2}=-1 / 8\right\}$. In $\bar{R} \backslash U$ we compare $u_{m}$ with the function $w$ defined by

$$
\begin{gathered}
w=\delta^{\prime} g(v), \quad v(x)=x_{2}-20 x_{1}^{2} \\
g(v)=e^{k v}-\mathrm{constant}, \quad g(-1 / 8)=0,
\end{gathered}
$$

for some $\delta^{\prime}, k$ to be chosen later.

Now notice that $D^{2} F_{m}$ is uniformly elliptic in $\mathcal{B}_{\rho}$ with ellipticity constants $\lambda, \Lambda$ depending only on $F$. Since

$$
D^{2} w=\delta^{\prime} e^{k v}\left(D^{2} v+k \nabla v \otimes \nabla v\right)
$$

we see that if the constant $\delta^{\prime}$ is chosen small enough so that $\nabla w \in \mathcal{B}_{\rho}$, and $k$ is sufficiently large depending only on $\lambda, \Lambda$ we have that

$$
\operatorname{Tr}\left(D^{2} F_{m} D^{2} w\right) \geq \lambda\left|\left(D^{2} w\right)^{+}\right|-\Lambda\left|\left(D^{2} w\right)^{-}\right|>0 .
$$

Therefore, $w$ is a subsolution and the minimum of $u_{m}-w$ must occur on $\partial(\bar{R} \backslash U)$.

Notice that by choosing $\delta^{\prime}$ possibly smaller depending on $\rho$ and $k$, we get that

$$
\alpha e_{2} \cdot x>w \text { on }\left\{x_{2}=1 / 8,\left|x_{1}\right| \leq 1 / 8\right\} \cup\left\{x_{1}= \pm 1 / 8, x_{2} \geq-\delta^{\prime \prime}\right\},
$$

and therefore

$$
u_{m}-w>0 \quad \text { on }\left\{x_{2}=1 / 8,\left|x_{1}\right| \leq 1 / 8\right\} \cup\left\{x_{1}= \pm 1 / 8, x_{2} \geq-2 \varepsilon / \rho\right\},
$$


for small $\varepsilon$. However, $u_{m}-w<0$ on $\partial U \cap\left\{x_{1}=0\right\}$. Hence the minimum must occur at some point $z_{0} \in \partial U \cap R$. Then,

$$
u_{m}(x) \geq w(x)-w\left(z_{0}\right), \quad x \in \bar{R} \backslash U .
$$

As in Proposition 6.4, this is a contradiction since the line $z_{0}+t e_{2}, t \geq 0$ intersects the other connected component of $\left\{u_{m}<0\right\}$, while on this line the function $w$ is increasing.

Proposition 6.3. Let $p_{0} \in \partial N$. Then, there exists $\varepsilon$ depending on $\delta, F$, such that if

then

$$
\left|u_{m}-p_{0} \cdot x\right| \leq \varepsilon, \quad x \in B_{1}
$$

$$
\left[\nabla u_{m}(x), p_{0}\right] \quad \text { is in a } \delta \text {-neighborhood of } \partial N, \text { for all } x \in B_{1 / 2} \text {. }
$$

Proof. We can argue as in the previous proof. Notice that in the proof of (8.1) and (8.2), we do not use that $p_{0} \in N$, thus they hold also for $p_{0} \in \partial N$. Then, when applying the localization Theorem, using also Proposition 5.2, we conclude that $\nabla u_{m}\left(B_{3 / 4}\right)$ is included in a $\delta$-neighborhood $\mathcal{N}_{\delta}$ of $\partial N$ if $\varepsilon$ is small depending only on $\delta, F$.

Again, it suffices to prove our conclusion at 0 , that is $\left[\nabla u_{m}(0), p_{0}\right] \subset \mathcal{N}_{4 \delta}$. By changing the system of coordinates in the $x$ and $p$ spaces, we can assume that we have the following situation:

$$
\begin{aligned}
& \nabla u_{m}\left(x_{0}\right)=0, \quad u_{m}\left(x_{0}\right)=0, \quad x_{0}= \pm e_{1} / 4, \quad p_{0}=\alpha e_{2} \\
& \left|u_{m}-\alpha e_{2} \cdot x\right| \leq 2 \varepsilon \quad \text { in } \bar{R}:=\left\{\left|x_{1}\right| \leq 1 / 8,\left|x_{2}\right| \leq 1 / 8\right\},
\end{aligned}
$$

and $\nabla u_{m}(\bar{R}) \subset \mathcal{N}_{\delta}$. We need to show that $\left[0, p_{0}\right] \subset \mathcal{N}_{4 \delta}$. We can assume that $\alpha>\delta$, otherwise the conclusion clearly holds since $p_{0} \in \partial N$. Moreover, the set $\left\{u_{m}<0\right\}$ has one connected component in $\bar{R}$ included in the strip $\left\{\left|x \cdot \alpha e_{2}\right| \leq 2 \varepsilon\right\}$ that intersects both sides $\left\{x_{1}= \pm 1 / 8\right\}$.

In the set $\bar{R} \backslash U$ (U as in the previous proof) we compare $u_{m}$ with the function $w$ given by

For small $\varepsilon$ we get

$$
w(x)=\frac{\alpha}{2}\left(x_{2}-20 x_{1}^{2}\right)+2 \varepsilon .
$$

$$
u_{m}-w>0 \text { on }\left\{x_{2}=1 / 8,\left|x_{1}\right| \leq 1 / 8\right\} \cup\left(\left\{x_{1}= \pm 1 / 8\right\} \cap(\bar{R} \backslash U)\right) .
$$

However, $u_{m}-w<0$ on $\partial U \cap\left\{x_{1}=0\right\}$. Hence the minimum of $u_{m}-w$ is negative and must occur at some point $z_{0} \in R \backslash U$. On the other hand, since $w$ is increasing in the $x_{2}$ direction, we obtain as in the previous proof that the minimum cannot occur on $R \cap \partial U$. Thus $z_{0}$ is an interior point and

$$
\nabla u_{m}\left(z_{0}\right)=\nabla w\left(z_{0}\right) .
$$

Moreover $\left|z_{0}\right| \leq \sqrt{\varepsilon / \alpha}$. Indeed, it is straightforward to check that if

$$
x \in\left\{\alpha x_{2} \geq-2 \varepsilon,|x| \geq \sqrt{\varepsilon / \alpha}\right\} \supset(\bar{R} \backslash U) \backslash B_{\sqrt{\varepsilon / \alpha}}(0),
$$

then

$$
u_{m}-w \geq \alpha x_{2}-w-2 \varepsilon \geq \varepsilon,
$$


D. DE SILVA AND O. SAVIN

and the claim follows. Therefore, as $\varepsilon \rightarrow 0, \nabla u_{m}\left(z_{0}\right) \rightarrow \alpha e_{2} / 2$. If $\varepsilon$ is small enough (depending on $\delta$ and $F$ ), $\nabla u_{m}\left(z_{0}\right) \in \mathcal{B}_{\delta}\left(p_{0} / 2\right)$ and $\nabla u_{m}\left(z_{0}\right) \in \mathcal{N}_{\delta}$ since $z_{0} \in \bar{R}$. Hence $p_{0} / 2 \in \mathcal{N}_{2 \delta}$ which implies $\left[0, p_{0}\right] \subset \mathcal{N}_{4 \delta}$, and the proposition is proved.

\section{REFERENCES}

[C] Caffarelli L.A., The obstacle problem revisited, J. Fourier Anal. Appl. 4 (1998), no. 4-5, 383-402.

[CFMS] Caffarelli L.A., Fabes E.B., Mortola S., Salsa S., Boundary behavior of nonnegative solutions of elliptic operators in divergence form, Indiana J. of Math., 30(1981), 621-640.

[CKP] Cohn H., Kenyon R., Propp J., A variational principle for domino tilings, J. Amer. Math. Soc. 14 (2001), no. 2. 297-346.

[D] De Giorgi E., Sulla differenziabilita' e l'analiticita' delle estremali degli integrali multipli regolari, Mem. Accad Sc. Torino, C. Sc. Fis. Mat. Natur. 3 (1957), 25-43.

[E] Evans, L. C. (1982). A New Proof of Local $C^{1, \alpha}$ Regularity for Solutions of Certain Degenerate Elliptic P.D.E., Journal of Differential Equations 45, 356-373.

[GT] Gilbarg D., Trudinger N. S., Elliptic partial differential equations of second order, Second edition. Grundlehren der Mathematischen Wissenschaften [Fundamental Principles of Mathematical Sciences], 224. Springer-Verlag, Berlin

[H] Hopf E., On S. Bernstein's Theorem on Surfaces $z(x, y)$ of Nonpositive Curvature, Proceedings of the American Mathematical Society, Vol. 1, No. 1 (Feb., 1950), pp. 80-85.

[KO] Kenyoyn R., Okounkov A., Limit shapes and the complex Burgers equation, Acta Math. 199 (2007), no. 2, 263-302.

[KOS] Kenyon R., Okounkov A., Sheffield S., Dimers and amoebae, Ann. of Math. (2) 163 (2006), no. 3, 1029-1056.

[LSW] Littmann W., Stampacchia G., Weinberger H.F., Regular points for elliptic equations with discontinuous coefficients, Ann. Scuola Norm. Sup. Pisa (3), 17 (1963), 43-77.

[M] Morrey C.B., Multiple Integral Problems in the Calculus of Variations and Related Topics, Univ. of California Publ. in Math., new ser. I, pp. 1-130, (1943).

[N] Nash J., Continuity of solutions of parabolic and elliptic equations, Amer. J. Math. 80 (1958), 931-954.

[S] Savin O., $C^{1}$ regularity for infinity harmonic functions in two dimensions, Arch. Ration. Mech. Anal. 176 (2005), no. 3, 351-361.

[Sc1] Schauder J., Uber lineare elliptische Differentialgleichungen zweiter Ordnung, Math. Z., 38 (1934), 257-282.

[Sc2] Schauder J.,Numerische Abschdtzungen in elliptischen linearen Differentialgleichungen, Studia Math., 5 (1934), 34-42.

[U] Uhlenbeck K., Regularity for a class of nonlinear elliptic systems,Acta Math., 138 (1977), $219-240$.

Department of Mathematics, Barnard College, Columbia University, New York, Ny 10027

E-mail address: desilva@math.columbia.edu

Department of Mathematics, Columbia University, New York, NY 10027

E-mail address: savin@math.columbia.edu 